\title{
ACUPUNTURA EN LA PREVENCIÓN, TRATAMIENTO DE LESIONES Y MEJORA DEL RENDIMIENTO EN LOS ATLETAS: REVISIÓN DE LA LITERATURE
}

\section{ARTÍCULO DE REVISIÓN}

JUNIOR, Julio Cesar Dias ${ }^{1}$

Junior, Julio Cesar Dias. Acupuntura en prevención, tratamiento de lesiones y mejora del rendimiento en atletas: Revisión de la literatura. Revista Científica Multidisciplinar Núcleo do Conhecimento. año 04, Ed. 10, Vol. 10, págs. 59-98. Octubre de 2019. ISSN: 2448-0959, Enlace de acceso: https://www.nucleodoconhecimento.com.br/salud/acupuntura-en-laprevencion

\section{RESUMEN}

Los estudios han demostrado que la acupuntura mejora el rendimiento físico en atletas profesionales, pero el gran problema son las lesiones deportivas, que pueden dejarlas lejos de las actividades durante largos períodos. El estudio llevó a cabo una revisión

${ }^{1}$ Licenciado en Fisioterapia por el Centro Universitario de Araraquara - UNIARA - 2005 (Araraquara-SP); Estudios de posgrado en Fisioterapia Ortopédica y Traumatológica del Instituto Cohen - Ortopedia, Rehabilitación y Medicina del Deporte - 2006 (Sao Paulo-SP)Formación en Terapia Manual Osteopática por el Instituto Cefisa - 2008 (Araraquara-SP); Mejora Profesional en la Reeducación Postural Global y Reprogramación Sensorial del Motor - RPG/RSM por el Instituto Paulista de Estudios Sistémicos - IPES - 2010 (Ribeir-o Preto-SP); Estudios de posgrado en Acupuntura Sistémica del Instituto Paulista de Estudios Sistémicos - IPES - 2013 (Ribeir-o PretoSP); Estricto grado sensu Máster en Desarrollo Territorial y Medio Ambiente del Centro Universitario de Araraquara - UNIARA - 2016 (Araraquara - SP); Mejora Profesional en Pilates Clínicos y Funcionales por el Instituto Paulista de Estudios Sistémicos IPES - 2016 (Ribeir-o Preto-SP). 
de la literatura, buscando la eficacia de la técnica en la prevención, mejora del rendimiento y en el tratamiento de lesiones. Algunos autores ejemplifican y aclaran el proceso de neurofisiología del dolor y la forma en que la acupuntura puede intervenir en este problema, otros, desarrollan y aplican protocolos para lograr una mejora en el rendimiento, el tratamiento de enfermedades y la prevención de Lesión. Entre los resultados se encontraron ochenta y nueve artículos sobre el tema, donde: veintidós informaron el tratamiento de las lesiones y treinta y siete la prevención y mejora del rendimiento deportivo, dirigiendo la aplicación una vez a la semana, de 20 - 30 minutos, presentando resultados satisfactorios para los datos analizados. Sobre la base de esta literatura y la práctica basada en la evidencia se puede concluir que la acupuntura es una gran intervención para lograr estos objetivos dentro del área del deporte.

Palabras clave: Acupuntura, lesiones, dolor, rendimiento.

\section{INTRODUCCIÓN}

Durante varios milenios la acupuntura se ha utilizado como una forma de prevención y tratamiento de diversas enfermedades del cuerpo. Esta técnica de la medicina china manipuló agujas de piedras y granos de pescado a lo largo de la edad de piedra (3000 años antes de Cristo). 1,2 El Emperador Amarillo Clásico: Huang Di Nei Jing, constituyó uno de los registros más antiguos de la Medicina Tradicional China (MTC), integrando hasta el día de hoy las bases filosóficas, que conceptualiza el equilibrio del universo y la naturaleza, que en su visión (china), la energía se llama Chi o Qi. Este factor tiene dos principios antagónicos que se completan y tienden a estar en equilibrio, a pesar de que está bajo la influencia del universo, llamado Yin y Yang. ${ }^{3}$

La acupuntura se define como la armonización del individuo con el entorno en el que se encuentra, no sólo, en insertar agujas y remediar el problema de la enfermedad, sino logrando el ideal de prevención de diversas patologías. ${ }^{4,5}$ Es un procedimiento realizado con objetivo terapéutico, no es tóxico, sin el uso de elementos químicos, con efectos secundarios mínimos, aunque es capaz de liberar sustancias endógenas como neurotransmisores. ${ }^{4,6,7,8,9}$ 
La intervención es estimular los acupuntos para lograr el efecto terapéutico deseado y lograr la homeostasis del organismo. Muchos estudios ejemplifican los efectos de la teoría neuronal no opioide (inhibe los impulsos nerviosos que actúan sobre el sistema nervioso periférico y central); teoría humoral (estimula la liberación de opioides endógenos, otras hormonas y neurotransmisores). ${ }^{10,11}$ Propósito en el control del dolor, la asociación de agujas con corriente eléctrica, llamada electroacuuntura, potencia y presenta beneficios en el práctica clínica, además de ser utilizado también como: acupuntura auricular; skullpuncture; moxabusto; ventosas; acupuntura láser. ${ }^{3 \text {, }}$ 10

En los deportes, el uso de la tecnología en busca de mejores resultados sigue creciendo, superando los límites del cuerpo humano. Varias terapias naturales o integrativas se estudian como una fuente de terapia complementaria para mejorar las condiciones atléticas. Aunque el número de estudios es escaso, la aceptación de la acupuntura sigue siendo muy curiosa, ya que muchos individuos todavía dudan de la evidencia científica. ${ }^{9}$ Sin embargo, su uso en el entorno deportivo ha ido en aumento, actuando en el tratamiento del dolor y las lesiones atléticas, acelerando el proceso de recuperación del individuo. Otro problema presentado es la intervención efectiva para mejorar el rendimiento físico y prevenir lesiones de atletas aficionados y profesionales $12,13,14$

\section{GOL}

El objetivo de este trabajo fue realizar una encuesta bibliográfica sobre el uso de la acupuntura en la prevención, la mejora del rendimiento deportivo y en el tratamiento de lesiones en diversas modalidades deportivas.

\section{METODOLOGÍA}

Se realizó una encuesta bibliográfica en las bases de datos Scielo, Google Acad-mico y Scoopus, para obtener información sobre: acupuntura en la prevención, mejora del rendimiento y en el tratamiento de lesiones deportivas. Se utilizaron las siguientes palabras clave: acupuntura; acupuntura y dolor; acupuntura en la prevención; lesión 
deportiva y acupuntura; acupuntura y mejora del rendimiento atlético; tratamiento de lesiones y acupuntura.

Para delimitar el recorte de este estudio, se realizó un cribado inicial entre los numerosos artículos encontrados, con un total de 89 manuscritos, seleccionando 22 que abordaron el tema de la acupuntura en el tratamiento de lesiones y 37 abordando la prevención de lesiones deportivas y mejorar el rendimiento deportivo. Sobre la base de este análisis, vale la pena mencionar el de todos los estudios encontrados principalmente, el universo de la investigación comprendía 59 artículos publicados en revistas científicas, fincas de congresos, libros, sitios web, que en el título, resumen o palabras clave terminologías utilizadas durante la investigación.

\section{RESULTADOS Y DISCUSIÓN}

En esta búsqueda dio lugar a varios artículos (Gráfico 1; Tabla 1), habiéndose realizado de forma aislada a través de las palabras clave.

Gráfico 1: Número total de artículos encontrados y utilizados en la búsqueda.

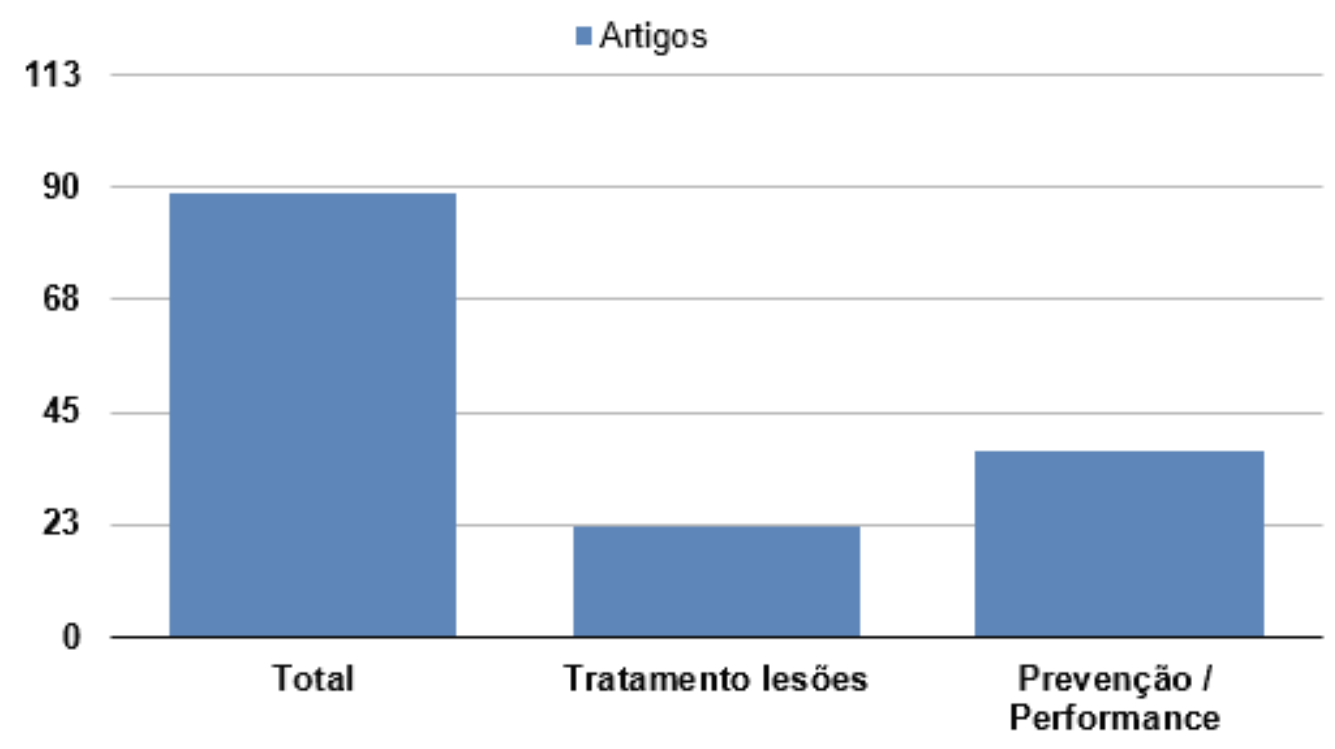


Tabla 1: Artículos relacionados con la prevención, la mejora del rendimiento deportivo y el tratamiento de lesiones deportivas.

\begin{tabular}{|c|c|c|}
\hline Autores & Título del artículo & Diarios/Anais/Congresos/Sitios \\
\hline $\begin{array}{l}\text { Ehrlich D, Haber } \\
\text { P, } 1992\end{array}$ & $\begin{array}{l}\text { Influence of } \\
\text { acupuncture on pysical } \\
\text { performance capacity } \\
\text { and Haemodynamic } \\
\text { Parameters }\end{array}$ & J. Sports Med \\
\hline $\begin{array}{lr}\text { Santos } & \text { VC, } \\
\text { Kawano } & \text { MM, } \\
\text { Banja RA, } 2008\end{array}$ & $\begin{array}{l}\text { Acupuntura na melhora } \\
\text { da performance em } \\
\text { atletas juvenis de } \\
\text { handebol }\end{array}$ & Rev Saúde e Pesq \\
\hline $\begin{array}{l}\text { Rubio K, Godoy } \\
\text { Moreira F, } 2008\end{array}$ & $\begin{array}{l}\text { A dor em corredores } \\
\text { com fascite plantar: o } \\
\text { uso da acupuntura }\end{array}$ & Rev Dor \\
\hline $\begin{array}{ll}\text { Pires } & \text { TF, } \\
\text { Pellegrinotti } & \text { IL, } \\
2010 & \end{array}$ & $\begin{array}{l}\text { Acupuntura na } \\
\text { Performance Atlética: } \\
\text { Estudo Exploratório }\end{array}$ & 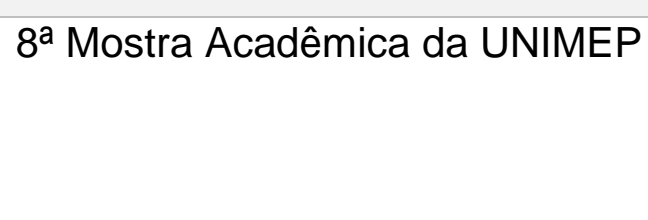 \\
\hline $\begin{array}{l}\text { Yang HY, Liu TY, } \\
\text { Gao M, } 2006\end{array}$ & $\begin{array}{l}\text { Electrical acupoint } \\
\text { stimulation increases } \\
\text { athletes rapid strength }\end{array}$ & Zhongguo Zhen Jiu \\
\hline $\begin{array}{ll}\text { Luna } & \text { MP, } \\
\text { Fernandes } & \text { Filho } \\
\text { J, } 2005 & \end{array}$ & $\begin{array}{l}\text { Efeitos da Acupuntura } \\
\text { na performance de } \\
\text { Atletas velocista de alto } \\
\text { rendimento do Rio de } \\
\text { Janeiro }\end{array}$ & Fit e Perform J \\
\hline $\begin{array}{l}\text { Dias Junior JC, } \\
\text { Marino DM, } 2019\end{array}$ & $\begin{array}{l}\text { Acupuntura na } \\
\text { prevenção de lesões } \\
\text { musculares em atletas } \\
\text { de futebol profissional }\end{array}$ & Rev Fisiot S Fun \\
\hline
\end{tabular}




\begin{tabular}{|c|c|c|}
\hline Maciocia G, 2007 & $\begin{array}{l}\text { Os fundamentos da } \\
\text { medicina chinesa: um } \\
\text { texto abrangente para } \\
\text { acupunturistas } \\
\text { fitoterapeutas }\end{array}$ & Libro \\
\hline $\begin{array}{ll}\text { Akimoto } & \text { T, } \\
\text { Nakahori } & \text { C, } \\
\text { Aizawa } & \text { K, } \\
\text { Kimura } & \text { F, } \\
\text { Fukubayashi } & \text { T, } \\
\text { Kono I, 2003 } & \end{array}$ & $\begin{array}{l}\text { Acupuncture and } \\
\text { responses of imunoligic } \\
\text { and endocrine markers } \\
\text { during competition }\end{array}$ & Med Sci in Sports Exerc \\
\hline $\begin{array}{ll}\text { Karvelas } & \text { BR, } \\
\text { Hoffman } & \text { MD, } \\
\text { Zeni Al, } 1996\end{array}$ & $\begin{array}{l}\text { Acute Effects of } \\
\text { Acupuncture on } \\
\text { Physiological and } \\
\text { Psychological } \\
\text { Responses to Cycle } \\
\text { Ergometry }\end{array}$ & Arch Phys Med Rehabil \\
\hline $\begin{array}{l}\text { Fry AC, Kraemer } \\
\text { KJ, } 1997\end{array}$ & $\begin{array}{l}\text { Resistance exercise ov } \\
\text { ertraing } \\
\text { and overreaching: neur } \\
\text { oendocrine responses }\end{array}$ & Sports Med \\
\hline KnardahI S, 1998 & $\begin{array}{l}\text { Sympathetic } \\
\text { nerve activity after } \\
\text { acupuncture in humans }\end{array}$ & $\begin{array}{l}\text { Department of } \\
\text { Neurophysiology }\end{array}$ \\
\hline $\begin{array}{l}\text { Barlas PJ, } \\
\text { Robinson JA, Ba } \\
\text { xter GD, } 2000\end{array}$ & $\begin{array}{l}\text { Lack ofeffect of acupun } \\
\text { cture upon signs } \\
\text { and symptoms of delay } \\
\text { ed } \\
\text { onset muscle soreness }\end{array}$ & Clinical Physio \\
\hline $\begin{array}{l}\text { Rossetto SC, } \\
2009\end{array}$ & $\begin{array}{l}\text { Acupuntura nos } \\
\text { Esportes }\end{array}$ & Libro \\
\hline
\end{tabular}




\begin{tabular}{|c|c|c|}
\hline $\begin{array}{l}\text { França D, } \\
\text { Fernandes- } \\
\text { Senna V, Cortez } \\
\text { CM, } 2004\end{array}$ &  & Fisioter Bras \\
\hline $\begin{array}{l}\text { Wadsworth L T, } \\
2006\end{array}$ & $\begin{array}{l}\text { Acupuncture in sports } \\
\text { medicine }\end{array}$ & Curr Sports Med Rep \\
\hline Barela J A, 2000 & $\begin{array}{l}\text { Estratégias de Controle } \\
\text { em Movimentos } \\
\text { Complexos: Ciclo } \\
\text { Percepção - Ação no } \\
\text { Controle Postural }\end{array}$ & Rev Paul de Educ Fís \\
\hline $\begin{array}{l}\text { Papler PG et al., } \\
1999\end{array}$ & $\begin{array}{l}\text { Reabilitação do joelho. } \\
\text { In: Greve J.M. A. e } \\
\text { Amattuzzi, } \\
\text { Medicina M.M. } \\
\text { reabilitação aplicada à } \\
\text { ortopedia } \\
\text { traumatologia }\end{array}$ & Libro \\
\hline $\begin{array}{l}\text { Gemeo LH. } \\
\text { Ignatti C, } 2004\end{array}$ & $\begin{array}{l}\text { Acupuntura como } \\
\text { Ferramenta Auxiliar do } \\
\text { Aumento da } \\
\text { Performance } \\
\text { Desportiva. In: Anais do } \\
\text { Simpósio Internacional } \\
\text { de Ciências Integradas } \\
\text { da UNAERP }\end{array}$ & Anais Simpósio \\
\hline
\end{tabular}




\begin{tabular}{|c|c|c|}
\hline Frasca L, 2011 & $\begin{array}{l}\text { Desempenho na Ponta } \\
\text { da Agulha }\end{array}$ & Rev Farmac \\
\hline Costa V, 2013 & $\begin{array}{lr}\text { Acupuntura } & \text { Previne } \\
\text { Lesões e ajuda a } & \\
\text { melhorar } & \text { o } \\
\text { Desempenho } & \text { nas } \\
\text { Pistas [periódico na } \\
\text { internet] }\end{array}$ & $\begin{array}{l}\text { http://blogs.oglobo.globo.com/pulso/ } \\
\text { post/acupuntura-previne-lesoes- } \\
\text { ajuda-melhorar-desempenho-nas- } \\
\text { pistas-500798.html. }\end{array}$ \\
\hline Luna M, 2016 & $\begin{array}{l}\text { Os Benefícios da } \\
\text { Acupuntura no Esporte }\end{array}$ & $\begin{array}{l}\text { http://www.ibramrp.com.br/noticia/49 } \\
\text { /os-beneficios-da-acupuntura-no- } \\
\text { esporte }\end{array}$ \\
\hline $\begin{array}{l}\text { Bopp-Limoge C, } \\
1998\end{array}$ & $\begin{array}{l}\text { L’acupuncture Permet- } \\
\text { elle D`ameliorer les } \\
\text { Performances } \\
\text { Sportives Stude } \\
\text { Personnelle a Propos } \\
\text { de } 35 \text { Sportifs de haut } \\
\text { niveau. }\end{array}$ & These Medicine \\
\hline Lin ZP et al., 2009 & $\begin{array}{l}\text { Effects of acupuncture } \\
\text { stimulation on recovery } \\
\text { ability for male elite } \\
\text { basketball athletes }\end{array}$ & Amer Jour of Chin Med \\
\hline $\begin{array}{l}\text { Lin, ZP et al., } \\
2009\end{array}$ & $\begin{array}{l}\text { Effect of auricular } \\
\text { acupuncture on oxygen } \\
\text { consumption of boxing } \\
\text { athletes }\end{array}$ & Chin Med Jour \\
\hline $\begin{array}{l}\text { Dhillon S et al., } \\
2008\end{array}$ & $\begin{array}{l}\text { The acute effect of } \\
\text { acupuncture on } 20-\mathrm{km} \\
\text { cycling performance. }\end{array}$ & Clin Jour of Spo Med \\
\hline $\begin{array}{l}\text { Hubscher M et } \\
\text { al., } 2010\end{array}$ & $\begin{array}{l}\text { Immediate effects of } \\
\text { acupuncture }\end{array}$ & Euro Jour of Appli Physi \\
\hline
\end{tabular}




\begin{tabular}{|c|c|c|}
\hline & $\begin{array}{l}\text { strength performance: a } \\
\text { ramdomized, controlled } \\
\text { crossover trial }\end{array}$ & \\
\hline $\begin{array}{l}\text { Geng } L \text { J et al., } \\
1995\end{array}$ & $\begin{array}{l}\text { Investigation on the } \\
\text { effects of ear } \\
\text { acupressure on } \\
\text { exercise-induced lactic } \\
\text { acid levels and the } \\
\text { implications for athletic } \\
\text { training }\end{array}$ & Amer Jour of Acupu \\
\hline Franco RS, 2012 & $\begin{array}{l}\text { Avaliação do Efeito da } \\
\text { Acupuntura sobre o } \\
\text { Desempenho Físico } \\
\text { pelo Teste do Banco de } \\
\text { Harvard }\end{array}$ & Dissertação \\
\hline $\begin{array}{l}\text { Fonseca LP, } \\
\text { Lessa JFM, } 2011\end{array}$ & $\begin{array}{l}\text { Efeito da Aplicação da } \\
\text { Acupuntura na } \\
\text { Resistência Muscular } \\
\text { Localizada de Membros } \\
\text { Superiores em } \\
\text { Praticantes de } \\
\text { Exercício Resistido. }\end{array}$ & Mobografia \\
\hline $\begin{array}{lr}\text { Belmiro } & \text { H, } \\
\text { Vicentini } & \text { D, } \\
\text { Camilotti } & \text { CM, } \\
2013 & \end{array}$ & $\begin{array}{l}\text { Efeitos da Acupuntura } \\
\text { no Desempenho Motor } \\
\text { de Atletas. }\end{array}$ & Rev Fac Educ Fis \\
\hline $\begin{array}{l}\text { Rubio K; Godoy } \\
\text { Moreira F, } 2007\end{array}$ & $\begin{array}{l}\text { A representação da dor } \\
\text { em atletas olímpicos } \\
\text { brasileiros }\end{array}$ & Rev Dor \\
\hline Parisotto D, 2014 & $\begin{array}{ll}\text { Efeito Imediato } & \mathrm{da} \\
\text { Aplicação } & \mathrm{da}\end{array}$ & Dissertação \\
\hline
\end{tabular}




\begin{tabular}{|c|c|c|}
\hline & $\begin{array}{l}\text { Acupuntura na Dor } \\
\text { Muscular Tardia e na } \\
\text { Capacidade de } \\
\text { Contração Muscular }\end{array}$ & \\
\hline Staud R, 2007 & $\begin{array}{l}\text { Mechanisms of } \\
\text { acupunture analgesia: } \\
\text { effective therapy for } \\
\text { musculoskeletal pain? }\end{array}$ & Curr rheumatol Rep \\
\hline $\begin{array}{l}\text { Bucinskaite V, } \\
\text { Lundeberg T, } \\
\text { Stenfors C, } \\
\text { Ekblom A, Dahlin } \\
\text { L, Theodorsson } \\
\text { E, } 1994\end{array}$ & $\begin{array}{l}\text { Effects of electro- } \\
\text { acupuncture and } \\
\text { pysical exercice on } \\
\text { regional concentrations } \\
\text { of neuropeptides in rat } \\
\text { brain }\end{array}$ & Brain research \\
\hline Shang C, 2009 & $\begin{array}{l}\text { Prospective tests on } \\
\text { biological models of } \\
\text { acupuncture }\end{array}$ & $\begin{array}{l}\text { Evid Based Complement Alternat } \\
\text { Med. }\end{array}$ \\
\hline Bowsher D,1988 & $\begin{array}{l}\text { Mechanisms of } \\
\text { acupuncture. In: Filshie } \\
\mathrm{J}, \text { White A. editors. } \\
\text { Medial Acupuncture: a } \\
\text { western scientific } \\
\text { approach. }\end{array}$ & Libro \\
\hline Ma SX, 2004 & $\begin{array}{lr}\text { Neurobiology } & \text { of } \\
\text { acupuncture: Toward } \\
\text { CAM }\end{array}$ & $\begin{array}{l}\text { Evid Based Complement Alternat } \\
\text { Med }\end{array}$ \\
\hline $\begin{array}{l}\text { Li J, Wang Q, } \\
\text { Liang H, Dong H, } \\
\text { Li Y, Ng EH, et al., } \\
2012\end{array}$ & $\begin{array}{l}\text { Biophysical } \\
\text { characteristics of } \\
\text { meridians and } \\
\text { acupoints: a systematic } \\
\text { review. }\end{array}$ & $\begin{array}{l}\text { Evid Based Complement Alternat } \\
\text { Med. }\end{array}$ \\
\hline
\end{tabular}


Lee, SH, Chung, Effects Acupunturaq on Neurocienses Letters

$\mathrm{SH}$, Lee, JS, Kim, the 5-

SS, Shin, HD, hydroxytryptamine

Lim, BV, et al., synthesis and

2002 tryptophan hydroxylase

expression in dorsal in

the dorsal raphe of

exercice rats.

Zyloney CE, Imaging the functional Mol pain

Jensen K, Polich connectivity of the

G, Loiotile RE, Periaqueductal Gray

Cheetham A, during genuine and

LaViolette PS, et sham

al., 2010 electroacupuncture treatment.

Minori AET, Mejia Atuação da Acupuntura Monografia

DPM, 2007 para o Tratamento de LER/DORT no Ombro.

$\begin{array}{lrlr}\text { Brum KN, } & \text { Tratamento } & \text { de } \\ \text { Alonso, AC, } & \text { massagem } & \text { e } \\ \text { Brech GC, 2009 } & \text { acupuntura } & \text { em } \\ & \text { corredoresrecreacionai } \\ & \text { s com síndrome do } \\ & \text { piriforme }\end{array}$

Hongwen S, 2003 Clinical Observation on J Tradit Chin Med.

Acupuncture Treatment of Piriformis Syndrome.

Rocha TBX, Análise Comparativa Rev Bras Cien e Mov

Vilela Junior GB, Eletromiográfica do

Martins GC, Reto Femoral em

Isometria na Posição 


\begin{tabular}{|c|c|c|}
\hline $\begin{array}{l}\text { Manzatto L, } \\
\text { Grande AJ, } 2012\end{array}$ & $\begin{array}{lr}\text { Inferior } & \text { do } \\
\text { Agachamento } & \text { Wall } \\
\text { Slide, antes e após a } \\
\text { Aplicação } & \text { da } \\
\text { Acupuntura no } & \text { Ponto } \\
\text { ST45 }\end{array}$ & \\
\hline $\begin{array}{l}\text { Pinheiro RG, } \\
\text { Mejia DPM, } 2012\end{array}$ & $\begin{array}{l}\text { Efeito da Acupuntura na } \\
\text { Melhora do Paciente } \\
\text { com Quadro Álgico de } \\
\text { Lesão de Menisco } \\
\text { Medial. }\end{array}$ & Monografia \\
\hline $\begin{array}{l}\text { Nunes EA, Mejia } \\
\text { DPM, } 2012\end{array}$ & $\begin{array}{lr}\text { Tratamento } & \text { de } \\
\text { Acupuntura } & \text { para } \\
\text { Combater Dores nos } \\
\text { Ombros. }\end{array}$ & Monografia \\
\hline Tolentino F, 2016 & $\begin{array}{l}\text { Efeito de um } \\
\text { Tratamento com } \\
\text { Auriculoterapia na Dor, } \\
\text { Funcionalidade } \\
\text { Mobilidade de Adultos } \\
\text { com Dor Lombar } \\
\text { Crônica. }\end{array}$ & Dissertação \\
\hline
\end{tabular}

\section{ACUPUNTURA EN LA PREVENCIÓN DE LESIONES}

La prevención de lesiones en el deporte es una gran batalla que ganan los profesionales que trabajan con atletas. En el fútbol, la lesión muscular corresponde al $20-40 \%$ de todas las lesiones deportivas, predominantemente $80-90 \%$ en las extremidades inferiores. ${ }^{15}$

La lesión muscular es el gran villano de todos los atletas, de varios deportes, y puede dejarlos alejados de sus actividades durante un largo período. Así, es interesante 
presentar una propuesta de intervención profiláctica, aportando la idea de una técnica para prevenir o reducir los riesgos de este tipo de lesiones en deportistas. En este caso la acupuntura resulta ser una técnica beneficiosa.

En su estudio, Dias Junior ${ }^{16}$, contó con la asistencia de 54 atletas masculinos, pertenecientes a un equipo de fútbol profesional. Se distribuyeron en 6 grupos: grupo 1 - acupuntura para reequilibrar el sistema, encontrados a través de la evaluación; grupo 2 - que recibió protocolo con puntos específicos: páncreas de bazo 3, páncreas de bazo 6 (Figura 1A) y estómago 36 (Figura 1B); grupo 3 - estómago 36 (Figura 1B); grupo 4 - páncreas del bazo 6 (Figura 1A); grupo 5 - acupuntura de oído; grupo de control - no recibió ninguna intervención. Después de ocho sesiones, una por semana, los resultados mostraron: los grupos 1 y 2 no presentaron ningún tipo de lesión; grupo 4: dos lesiones; grupos 3 y 5: se presenta 1 lesión; control: presentó 6 casos de problemas musculares. 
Figura 1 A: páncreas del bazo 3 - en el lado medial del pie, postteroinferior de la articulación metatarsal-falina, en la línea de la unión de la piel oscura y clara; páncreas bazo 6: 3 tsun por encima del maleol medial, en el borde posteromedial de la tibia; B: estómago 36 - 3 tsun por debajo de la rótula entre el músculo de la tibia anterior y el largo músculo extensor de los dedos; estómago 37 - 3 tsun por debajo de Zusanli (E36), en el lado lateral anterior del músculo tibia; estómago 38 - 8 tsun por debajo de la rodilla, 2 tun por debajo del Point Shangjuxu (E37), en el lado del músculo de la tibia anterior; estómago 41 - en el punto medio dorsal del tobillo por encima del ligamento cruzado, entre los tendones del largo músculo extensor hallux y el extensor largo de los dedos.

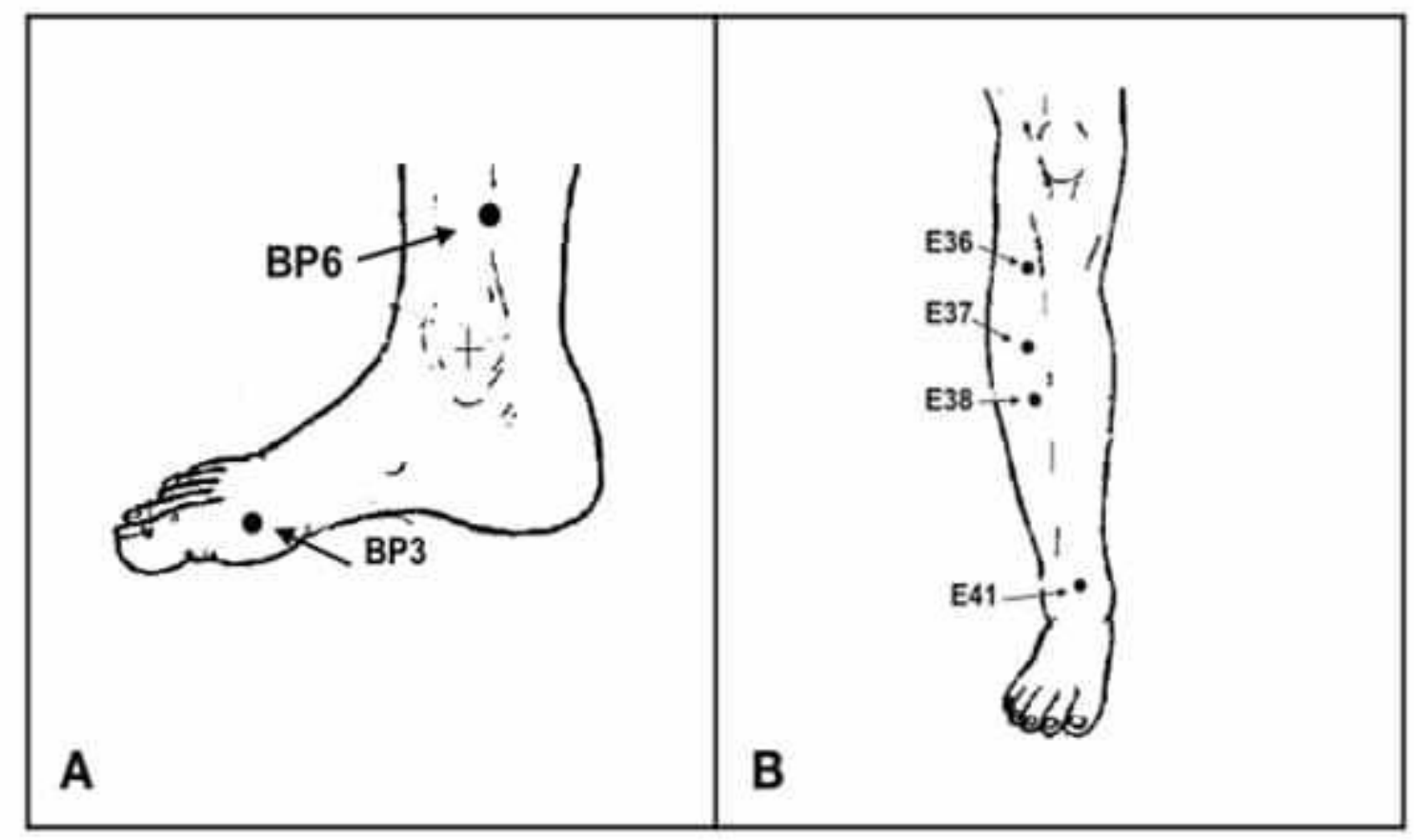

Fuente: Wen ${ }^{17}$

El bazo del páncreas tiene la función de extraer energía de los alimentos, distribuir al cuerpo y controlar la sangre dentro de los vasos sanguíneos. El Qi de presentación fuerte será transportado a todos los músculos del cuerpo, específicamente a las extremidades, pero si el Qi está en deficiencia, la energía no se transmitirá al tejido muscular y el atleta presentará fatiga y músculos debilitados. ${ }^{18}$ 
Según Ross ${ }^{19}$, los puntos del bazo páncreas 3 y 6 están asociados con el estómago 36 por tonificar la sangre Qi. El elemento de la tierra es responsable del equilibrio energético y la disponibilidad de Qi y la sangre como fuentes de energía para la actividad mental, emocional y física. EL CCM, informa que mediante la tonifión de este elemento, mantiene los músculos nutridos por Qi y Xue (sangre en el CCM), con el objetivo de mejorar el rendimiento atlético. Esto mantiene la obligación de tonificar el órgano que produce Xue (Hígado) y mantiene el líquido energético (Páncreas de bazo y riñón), ya que la funcionalidad de la sangre es nutrir el cuerpo, además de completar la acción nutritiva de Qi.

Entrenamiento muscular, relajación y fuerza contráctela está relacionada con la nutrición del hígado xue. Si la nutrición es deficiente, los calambres y la parestesia de las extremidades causarán lesiones. Por otro lado el bazo monitorea el xue dentro de los vasos y elimina el qi de la transmisión de alimentos al cuerpo: Qi fuerte, transmitirá buena energía a los músculos; un Qi debilitado resultará en un músculo cansado y débil. ${ }^{19,20}$

Sobre la base de esta evidencia esta técnica se practica en deportes presentando resultados rápidos, mejorando la calidad de la fuerza muscular, condiciones cardiorrespiratorias, flexibilidad y bienestar mental y físico de los deportistas. ${ }^{20,} 21,22$ Yang y 22 empleados, lograron un aumento significativo en la fuerza muscular, proporcionando una mejora en la velocidad de los atletas. A su vez, Luna y los colaboradores ${ }^{24}$, afirmaron que la estimulación de los puntos de acupuntura mejora el rendimiento deportivo, la plasticidad muscular evitando lesiones.

Un estudio realizado en Japón destaca el efecto de la acupuntura en el bienestar físico de las jugadoras de fútbol durante la competición. Se dividieron en dos grupos, donde uno fue sometido a tratamiento y el otro control. Los niveles de cortisol (evaluaciones del sistema endocrino) y la evaluación del bienestar físico se evaluaron utilizando el cuestionario POMS (evalua el estado físico y mental): se observó una mejora en el sistema inmunitario ( disminución de la secreción de SIgA), mejora del estrés mental (disminución del cortisol) y mejora el bienestar físico, la flexibilidad y la tensión muscular. ${ }^{25}$ 
Sin embargo Ehrlich y 25 empleados ' lograron una mejora en el rendimiento deportivo, aumentando el nivel de bienestar físico, mejorando la respuesta competitiva, durante el entrenamiento y los juegos, ayudando en la recuperación de la capacidad muscular.

Otras lesiones que se pueden prevenir con la acupuntura son los esguinces de tobillo, que afectan los ligamentos, desencadenando muchos dolores y la limitación de la función articular. France ${ }^{26}$, utilizó los puntos ashi (puntos dolorosos a la palpación), asociados con la vejiga 60 (Figura 2A) para eliminar el dolor; la vesícula biliar 39 que refuerza los huesos, tendones y lucha contra el algia en las extremidades; y la vesícula biliar 40 estimula el Qi y el xue; estómago 41 (Figura 2B) que trata los cambios laterales del tobillo y el estómago 36 (Figura 1B) eliminando la obstrucción y estimulando la circulación de Qi y xue local.

Figura 2 A: vejiga 60 - entre el tendón de Aquiles y el borde del solelono lateral del tobillo, en el nivel del punto más alto del maleol; B: vesícula biliar 39 - 3 tsun por encima del punto más alto del malémalo externo, en depresión entre el peroné y tendones fibulares largos y cortos y la vesícula biliar 40 - en el lado antero-inferior del maléolo externo, en la depresión lateral del tendón del largo músculo extensor digital ; estómago 41 - en el punto medio dorsal del tobillo por encima del ligamento cruzado, entre los tendones del largo músculo extensor halux y el extensor largo de los dedos.

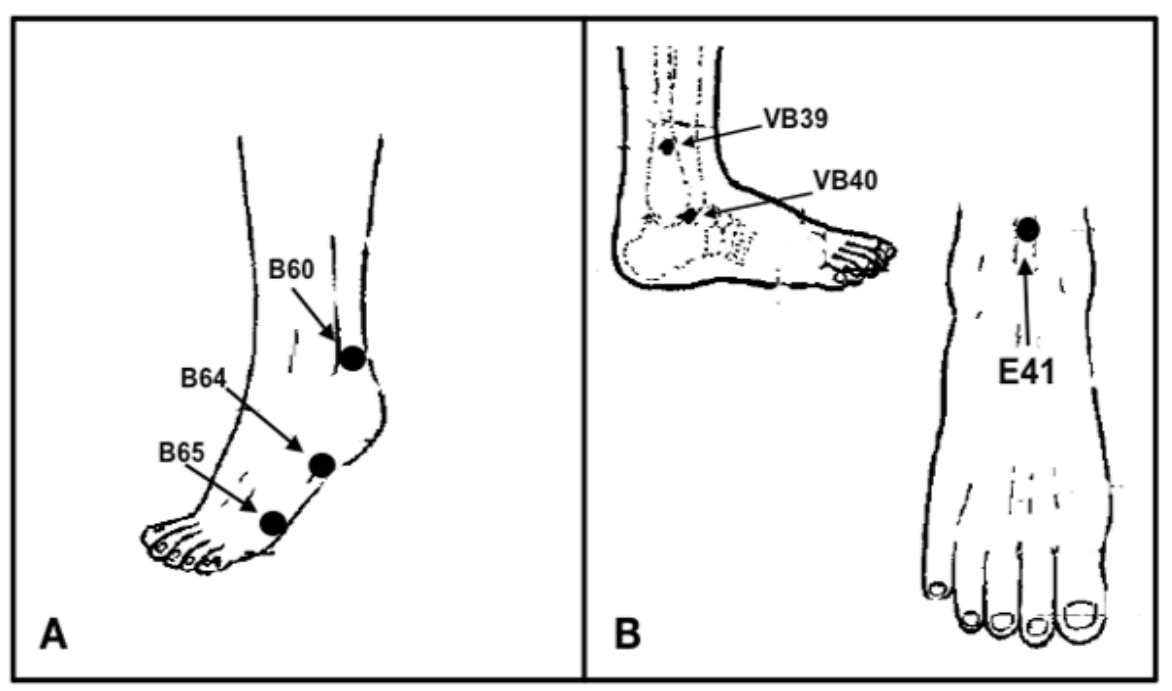

Fuente: Wen ${ }^{17}$ 
Algunos atletas pueden sorprenderse con espraincolor, que se define como una lesión del tendón cervical que causa dolor muscular y rigidez. Hay un protocolo para remediar este problema y devolver al atleta a sus deberes. Wadsworth ${ }^{27}$, utilizado además de los puntos ashi que estimulan la circulación de Qi y xue, la vesícula biliar 39 (Figura 2B), la vesícula biliar 20 actúa en la circulación de Qi y xue, relajando tendones y suavizando el dolor; gobernador ousse 14 expulsando el factor patógeno y regulando el Meridiano Qi; vejiga 10; intestino delgado 14; la vesícula biliar 21 (Figura 3A) estimula naífilos de Qi y xue. Si el bloqueo articular limita la flexión y la extensión, utilice el punto de vejiga 60 (Figura 2A). Los puntos 3 del intestino delgado, el pulmón 7 promueven la circulación del Qi y el xue del cuello, si tiene limitación de rotación, utilice el punto 7 del intestino delgado que drena el meridiano Taiyang de la mano (Figura 3B). 
Figura 3 A: vesícula biliar 20 - por debajo del borde occipital en la depresión entre los músculos trapezoide o esternón-clidomastoideo, en el margen del cabello y la vesícula biliar 21 - en el punto equícula entre el Dazhui (VC14) y el acromion del hombro, 1 tsun por encima del punto de Tianilian; jarrón del gobernador 14 - en el punto medio entre los procesos espinosos; séptima vértebra cervical y primera vértebra dorsal; vejiga 10 - a nivel entre las espinas de la segunda y tercera vértebras, 1,3 tun lateral de la línea media dorsal, en el lado del borde del músculo trapecio y el intestino delgado 14 - 3 tun lateral del eje de la vértebra; en el nivel horizontal del borde inferior del proceso espinoso de la primera vértebra dorsal; B: pulmón 7 - en el lado medial del antebrazo, 1,5 tsun por encima de la línea de la muñeca entre los tendones del músculo aductor pulgar largo y el largo músculo extensor carporadial; intestino delgado 3 - lado cubital de la mano, detrás de la articulación metacarpal-falange del quinto dedo, entre las pieles claras y oscuras; Intestino delgado 7 - 5 tsun por encima de la muñeca, en el lado cubital del músculo extensor carpal-ulnar.

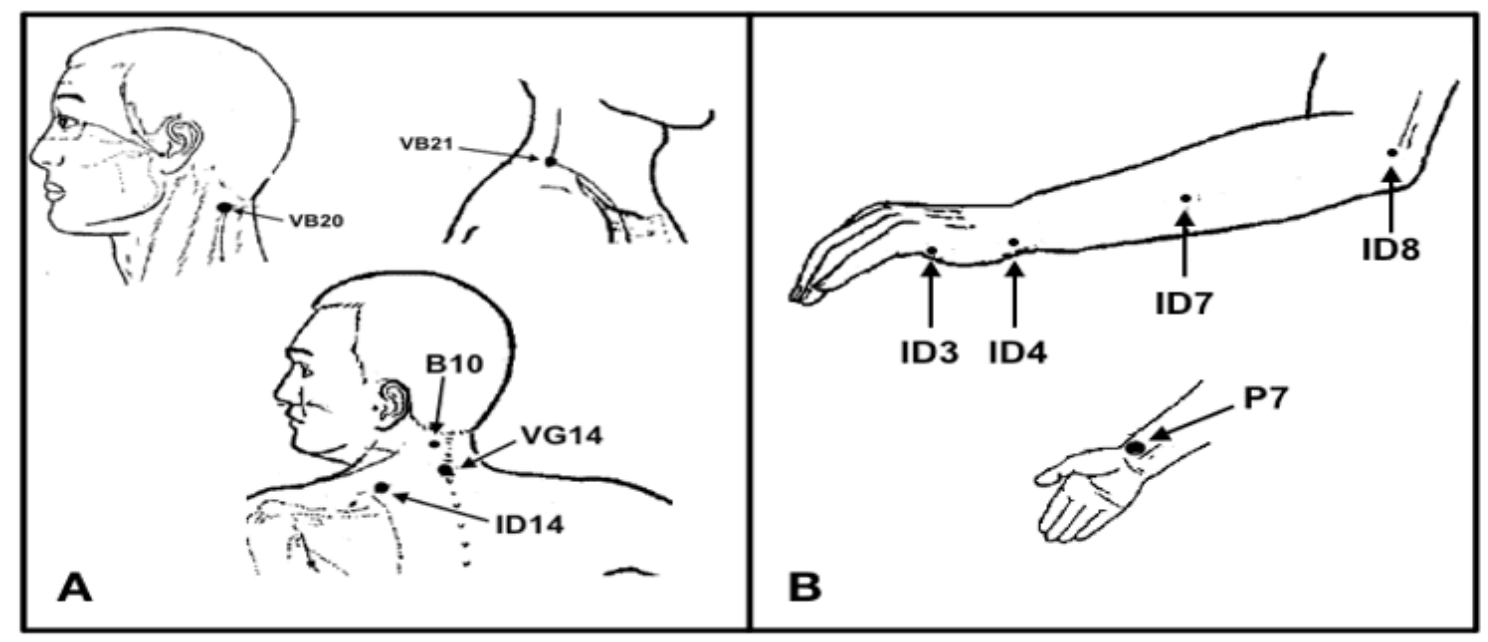

Fuente: Wen ${ }^{17}$

Sobrecargar la articulación del codo, afecta, especialmente a los jugadores de tenis, y puede conducir a un bloqueo de Qi y xue, desencadenando una inflamación crónica llamada epicondilite. Los puntos Ashi se utilizan en la región que promueve la estimulación Qi. ${ }^{28}$ 
Para el tratamiento de lesiones de muñeca, tales como: tendinitis o tenosinovitis del pulgar, extensores y flexores de los dedos' Francia 26, utilizó el punto ashi asociado con el intestino grueso 5 para dispersar el calor del meridiano; intestino delgado 5 eliminando el calor y desbloqueando la muñeca (Figura 4A); Calentador triple 4 que además de eliminar el calor cesa la inflamación y el intestino grueso 11 (Figura 4B) desbloquea qi y xue calmando el dolor.

Figura 4 A: intestino delgado 5 - en el lado cubital de la muñeca, en la depresión entre el proceso pisiforme y el estiloide cubital; Intestino grueso 5 - en el lado radial posterior de la muñeca, un poco distal del hueso de la radio, donde hay depresión entre los tendones del músculo extensor corto y largo del pulgar, al estirar y abrir el pulgar. B: calentador triple 4 - en el lado dorsal de la muñeca, en la depresión en el medio del pliegue dorsal de la muñeca, entre los tendones de los músculos extensores digitales comunes y extensor digital del quinto dedo;intestino grande 11 - en el lado radial del codo, en el músculo braquioradial; al doblar el codo, en depresión radial al final de la línea cubital.

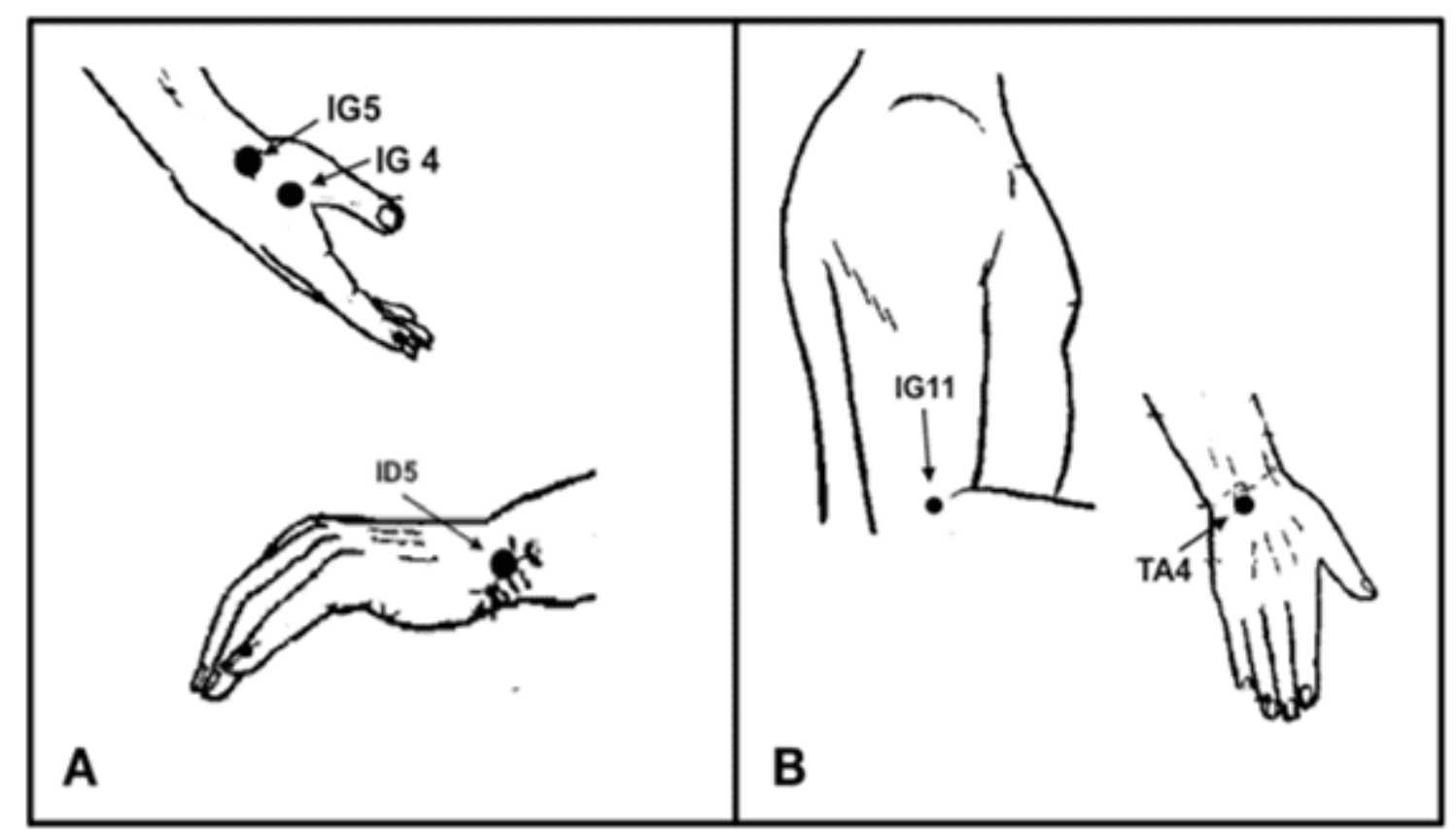

Fuente: Wen ${ }^{17}$ 
Para el dolor de espalda baja, Barela ${ }^{17}$, puntos ashi asociados con la vejiga 23 y la vejiga 25 para estimular el Qi y Xue locales, vejiga 40 (Figura 5) que elimina el dolor y el calor.

Figura 5: vejiga 23 - 1.5 tsun, lateral del borde inferior del proceso espinal de la vértebra (L2); vejiga 25 - 1.5 tsun, lateral del borde inferior del proceso espinal de la vértebra (L4); vejiga 40 - 3 tsun, lateral del eje (Du-Mai), en el nivel del borde inferior en la segunda columna vertebral de la vértebra (T2).



Fuente: Wen ${ }^{17}$

Ahora otra lesión que preocupa a los atletas de fútbol, son ligamentos, meniscales y tendones de la rodilla. Esto se trata con puntos de ashi, asociados con la vesícula biliar 33 (Figura 6) que relaja los tendones y trata el dolor; la vejiga 40 (Figura 5) elimina el dolor articular, el edema y el calor, estimulando qi y xue; estómago 36 (Figura 1B) que regula el flujo de Qi y xue del meridiano eliminando la obstrucción. ${ }^{29}$ 
Figura 6: vesícula biliar 33 - en el borde lateral de la rodilla, 3 tsun por encima del punto yanglingquan (VB34), en la depresión superior del epitenodilof lateral del fémur.

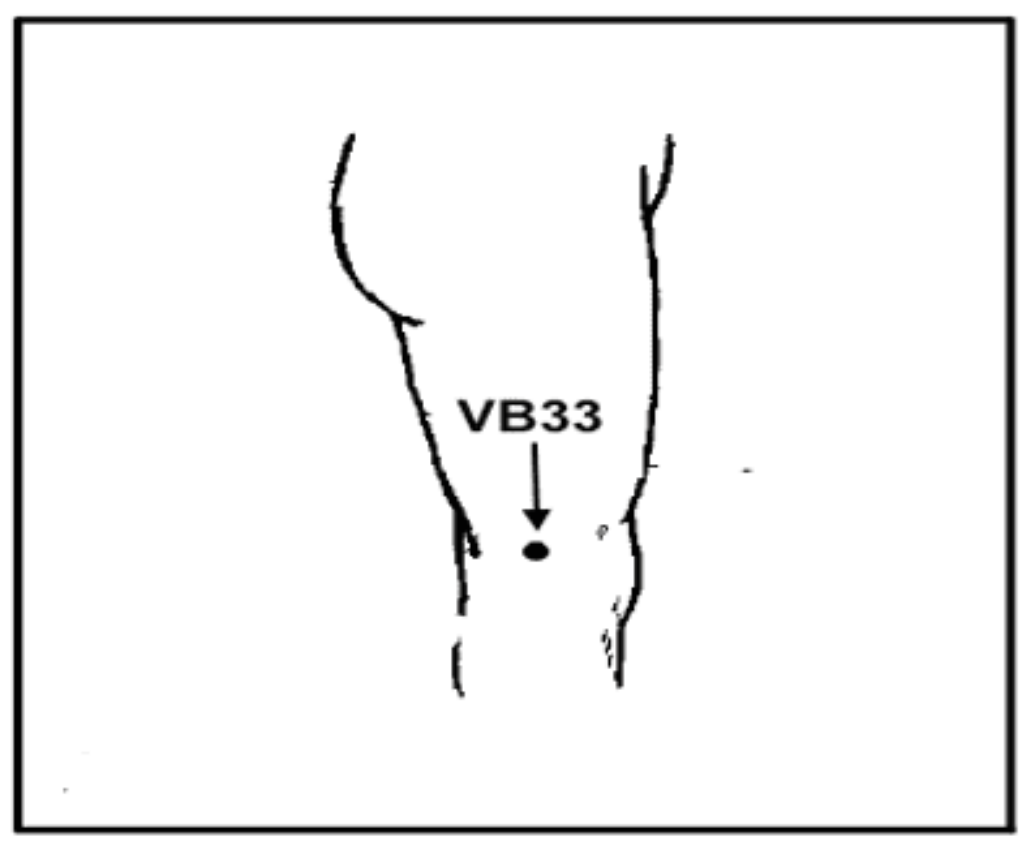

Fuente: Wen ${ }^{17}$

Gemeo ${ }^{30}$, declaró que la acupuntura puede promover una mejora en la capacidad física de los atletas, prevenir y tratar lesiones que pueden ocurrir antes, durante o después de las competiciones deportivas, además de ayudar e influir en factores emocionales como la ansiedad, irritabilidad, insomnio y depresión, que puede obstaculizar directamente la capacidad atlética de estos individuos. Desarrolló un protocolo específico utilizando puntos de acupuntura maestro, seleccionando de acuerdo a la modalidad (Gráfico 1):

Tabla 2: Gemeo ${ }^{30}$

\section{Maestro de la energía jarrón regulador 19 (Figura 7) moral}




\begin{tabular}{|l|l|}
\hline $\begin{array}{l}\text { Maestro de energía } \\
\text { general }\end{array}$ & $\begin{array}{l}\text { influye en la energía general, actuando sobre la } \\
\text { energía ancestral y postnatal - jarrón del gobernador } \\
4,6,10,13 \text { (Figura 7) }\end{array}$ \\
$\begin{array}{ll}\text { Maestros las } \\
\text { extremidades superiores }\end{array}$ & $\begin{array}{l}\text { actúa sobre los movimientos del antebrazo, muñecas, } \\
\text { manos y hombros: triple calentador 15 (Figura 7) }\end{array}$ \\
\hline $\begin{array}{l}\text { Maestro de los pulmones } \\
\text { interfiere en el tórax anterior: pulmón 1 (Figura 7) }\end{array}$ \\
\hline Back master & $\begin{array}{l}\text { diafragma, respiración y nn. fénico: vejiga17 (Figura } \\
7 \text { ) }\end{array}$ \\
\hline Maestro del vientre & $\begin{array}{l}\text { influencia en la cintura abdominal: estómago 27 } \\
\text { (Figura 7) }\end{array}$ \\
\hline Maestro de los riñones & $\begin{array}{l}\text { actos de la cintura lumbar: vejiga 47 y vejiga 52 } \\
\text { (Figura 8) }\end{array}$ \\
\hline Maestro muslo & $\begin{array}{l}\text { actúa el mm. glúteos y coxofemoral (Figura 8) } \\
\text { actúa en la musculatura de los muslos: vesícula biliar } \\
\text { 30 (Figura 8) }\end{array}$ \\
\hline $\begin{array}{l}\text { Maestro de miembros } \\
\text { inferiores }\end{array}$ & $\begin{array}{l}\text { influye en la acción de los pies y los músculos en } \\
\text { general: vejiga 58 (Figura 8) y estómago 36 (Figura } \\
\text { 1B) }\end{array}$ \\
\hline $\begin{array}{l}\text { Puntos para mejorar el } \\
\text { equilibrio y la reflexión }\end{array}$ & $\begin{array}{l}\text { vaso concepción 4, vaso concepción 6 (Figura 9), } \\
\text { intestino grueso 11 (Figura 4B), vejiga 46 (Figura 8), } \\
\text { vaso 4 (Figura 7). }\end{array}$ \\
\hline
\end{tabular}

Figura 7: jarrón del gobernador 4 - en la línea central de la columna vertebral de la columna vertebral; en el espacio entre las espinas de la segunda y tercera vértebras lumbares; jarrón del gobernador 6 - en la línea central de la columna; entre el undécimo y duodécimo proceso espinoso de las vértebras dorsales; jarrón regulador 10 - en la línea central de la columna; entre el sexto y séptimo proceso espinoso de las vértebras dorsales; jarrón del gobernador 13 - en la línea central de la columna; entre el primer y segundo proceso espinoso de las vértebras dorsales; jarrón del gobernador 19 - 1.5 tsun detrás de Baihui (VG 20); estómago 27 - 2 tsun por debajo del ombligo y 2 tsun junto a la línea medial en el borde lateral del músculo rectoabdominal; vejiga 17 - 1.5 tsun del eje, en el nivel del borde inferior del proceso espinal 
de la vértebra (T7); pulmón 1 - en el lado anterolateral del pecho, por debajo del punto Yunmen (P2) (agujero entre la clavícula y el hombro), en el espacio entre la primera y la segunda costilla, 6 tsun en el lado de la línea media del cuerpo; triple calentador 15 - en el pozo supraescapular, entre Quyuan (ID13) y Jianjing (VB21).

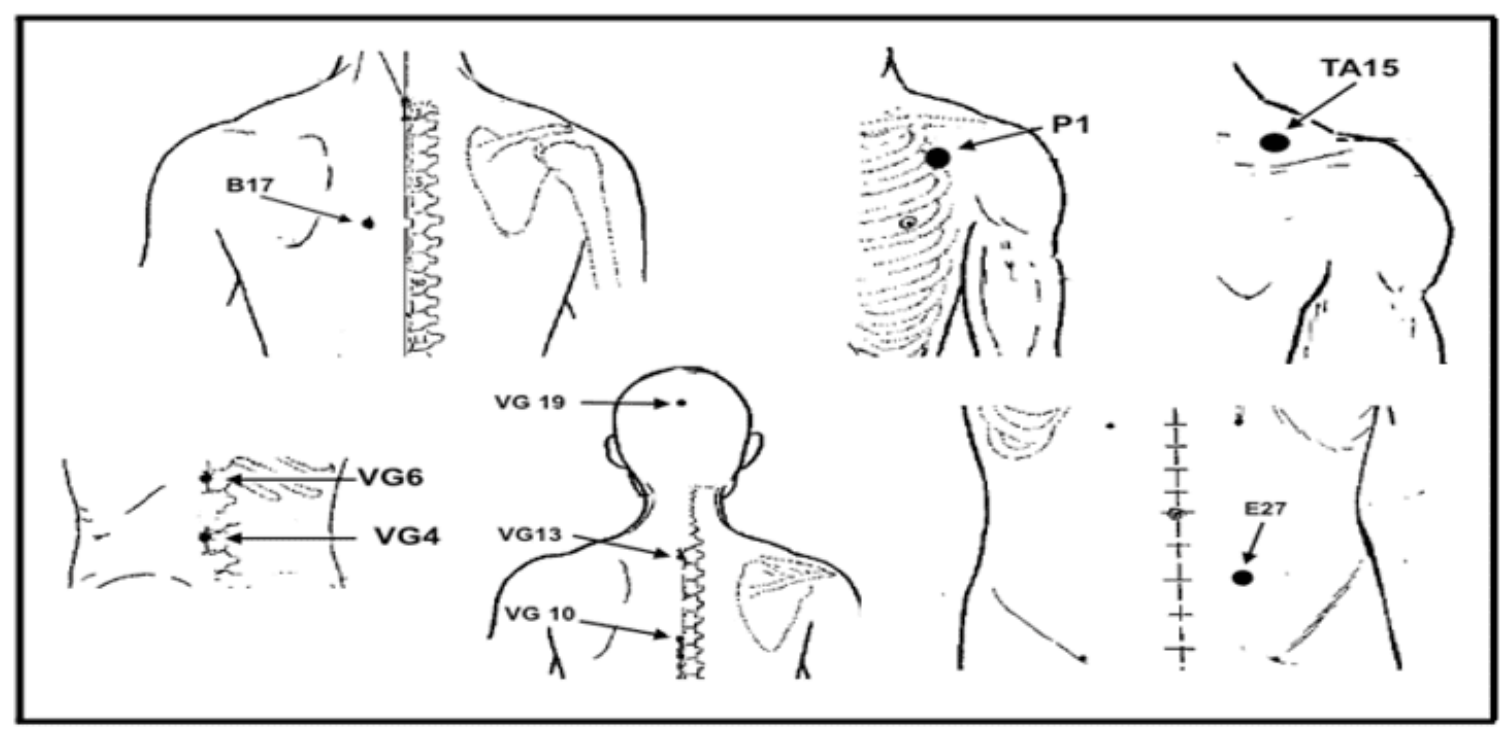

Fuente: Wen ${ }^{17}$ 
Figura 8: vejiga 46 - 3 tsun, lateral del eje, en el nivel del borde inferior de la columna vertebral de la vértebra (T9); vejiga 47 - 3 tsun, lateral del eje, en el nivel del borde inferior de la columna vertebral de la vértebra (T10); vejiga 52 - 3 tsun, lateral del eje, a nivel de la segunda columna vertebral del sacro, en el borde lateral de la articulación iliossacral; vejiga 58 - I tsun por debajo del lado del punto de chengshan (B57); 7 tsun por encima del talón, en el lado lateral del tendón muscular gastrocnemius; vesícula biliar 30 - en el glúteo, en la línea entre el hiato sagrado y el prominente del trocánter más grande; un tercio de la distancia lateral en el borde inferior del músculo piriforo; punto de cadera adicional - en el centro de la línea que van desde el extremo superior de la ranura intergluteal a la parte de la cresta exterior del isquio.

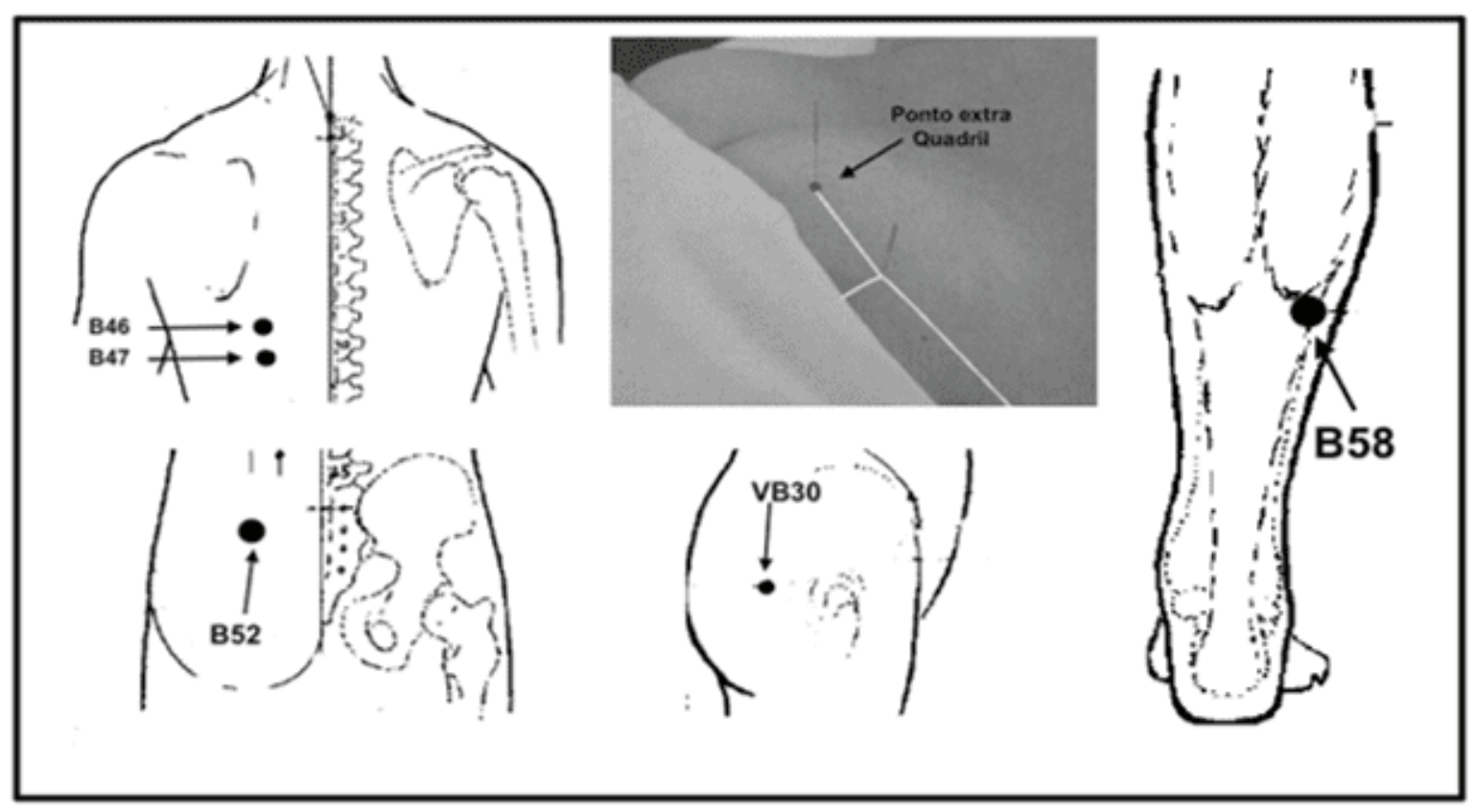

Fuente: Wen ${ }^{17}$; Rosseto ${ }^{31}$ 
Figura 9: concepción del jarrón 4 - 3 tsun debajo del ombligo, en la línea central del abdomen; concepción del jarrón 6 - 1.5 tsun por debajo del ombligo, en la línea central del abdomen.

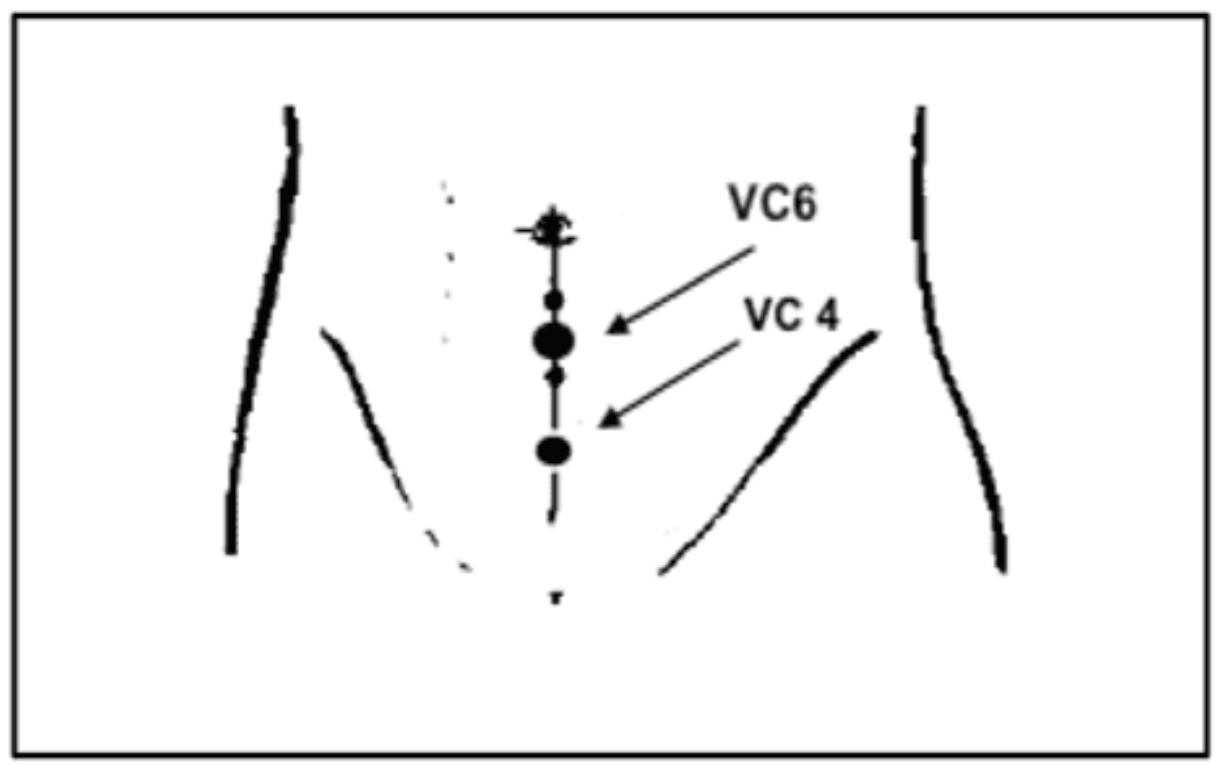

Fuente: Wen ${ }^{17}$

\section{ACUPUNTURA EN LA MEJORA DEL RENDIMIENTO ATLÉTICO}

Cuando se trata de la acupuntura en la prevención de lesiones en atletas, no hay que olvidar que el logro de este objetivo preventivo, por lo tanto mejoramos la calidad y la integridad física y podemos intervenir en la mejora del rendimiento.

En los países asiáticos el uso de la acupuntura desarrolla un bienestar físico para los atletas, regulado por los sistemas nervioso, endocrino, inmune y como resultado, una mejora en la condición física en varias modalidades. ${ }^{32,31,34}$ Además hay puntos aplicados antes, durante y después del entrenamiento y los juegos, que ayudan en el mantenimiento de los músculos, como reducir el ácido láctico, reducir el dolor, la fatiga, acelerar la recuperación y mejorar el rendimiento. ${ }^{35}$

La acupuntura estimula la liberación de sustancias bioquímicas que alivian la fatiga muscular de los ejercicios intensos, además de promover el aumento de la actividad antioxidante, disminuyendo las tasas de estrés oxidativo. Durante un estudio, los 
atletas recibieron tratamiento con electroacupuntura durante 30 minutos, durante un período de 30 días y el resultado fue el aumento significativo de la superóxido dismutasa (SOD) y la disminución de la malondial en sangre (MDA). Superóxido dismutasa es una enzima con efectos antioxidantes que protege las células contra sustancias tóxicas mediante el ejercicio de una potente respuesta antiinflamatoria en el cuerpo. EI MDA está relacionado con enfermedades inflamatorias y degenerativas, lo que permite a los investigadores concluir que la electroacupuntura disminuye las tasas de MDA y aumenta la SOD, contribuyendo al alivio de la fatiga y mejorando el rendimiento físico. ${ }^{36}$

Aunque es una técnica milenaria, su uso mejora el rendimiento físico y mental del atleta es reciente. Luna, publicó una investigación que demuestra que la acupuntura mejoró estadísticamente la máxima fuerza y potencia de los atletas velocistas (100, 200 y 400 metros poco profundo sin barreras) y desde entonces la investigación en este sentido se ha profundizado. ${ }^{37}$

En una investigación, Santos 20 utilizó atletas masculinos de Handbol entre 15 y 17 años de edad, donde realizó dos tiros de 100 metros en cada atleta (pre acupuntura). Después de 45 minutos de aplicación del protocolo elegido para el tratamiento, reevaluó de la misma manera. Los puntos elegidos fueron: vejiga 58 (Figura 8) maestro de las piernas y el pie; estómago 36 (Figura 1B) tonifica la musculatura estriada y se utiliza para potenciar el tratamiento; punto maestro adicional de las caderas (Figura 8) tonifica los glúteos y aumenta la flexibilidad de la articulación coxofemoral; vesícula biliar 30 (Figura 8) maestro de los muslos y promueve la flexibilidad para el movimiento de las extremidades inferiores; pulmón 1 (Figura 7) maestro de los pulmones promueve el trabajo respiratorio eficiente; vejiga 17 (Figura 7) maestro de la espalda, diafragma dorsal shu y punto de influencia de Xue que regula el ritmo respiratorio; triple calentador 15 (Figura 7) maestro de los brazos que manda el grupo muscular articular de hombros, nuca, columna cervical, primeras seis vértebras torácicas, brazos, antebrazos, muñecas y mano.

Los resultados identificaron una mejora significativa, con una disminución en el tiempo en la segunda batería de las pruebas de 100 metros. La mejora media en los tiempos 
fue del $5,72 \%$ y el atleta con el mejor rendimiento alcanzado alcanzó un porcentaje del $10,84 \%$.

Este promedio de 5,72\% logrado en la mejora del rendimiento parece pequeño, pero si se compara con los resultados de competiciones importantes, podemos ver la validez de la práctica: en los Juegos Panamericanos de Río de Janeiro 2007, en la final de los 100 metros poco profundos macho a la diferencia entre el 1o lugar y el octavo fue 0.23 segundos, lo que representa un tiempo de $2.26 \%$ más alto, es decir, si este atleta lograra una mejora de $5.72 \%$ en su rendimiento, probablemente recibiría otros resultados.

Hay poca atención científica, cuando tratamos, de los efectos de la acupuntura en las mejoras en la respuesta fisiológica inducida por el ejercicio y pocos dirigidos a atletas de alto rendimiento. ${ }^{24,38}$ Pero Rossetto ${ }^{31}$ presenta un protocolo para mejorar el rendimiento deportivo en fútbol y rugby utilizando los siguientes puntos: vejiga 52 y vejiga 58 (Figura 8); vejiga 17 y pulmón 1 (figura 7).

El punto 58 de la vejiga, tonifica la musculatura estriada de todo el cuerpo, articulación de las rodillas, tobillos y pies. Es un punto importante para evitar la fatiga, especialmente si se utiliza con el estómago 36 . La vejiga 17 tonifica los músculos dorsales, los ligamentos articulares e incluso aumenta la flexibilidad de las propias articulaciones vertebrales, promoviendo la acción reguladora en el diafragma, el ritmo respiratorio y los nervios frenic. La vejiga 52 tonifica toda la región de los músculos lumbares y promueve la fuerza de voluntad. Pulmón 1 se utiliza para hacer el trabajo respiratorio eficiente y está relacionado con los nervios supraclaviculares e intercostales (con inserción más profunda) ayudando en el plexo braquial. El uso con el punto de vejiga 17 regulariza el ritmo respiratorio.

Al igual que Bopp Limoge 39, aplicó un protocolo fijo de tres puntos: hígado 5 (Figura 10), calentador triple 15 (Figura 7) y vejiga 58 (Figura 8), añadido a otros dos puntos aleatorios, placebo, en 35 atletas, logrando una mejora significativa en el rendimiento atlético de los individuos probados. 
Figura 10: hígado 5 - 5 tsun por encima del maléolo medial; en el borde posteromedial de la tibia.

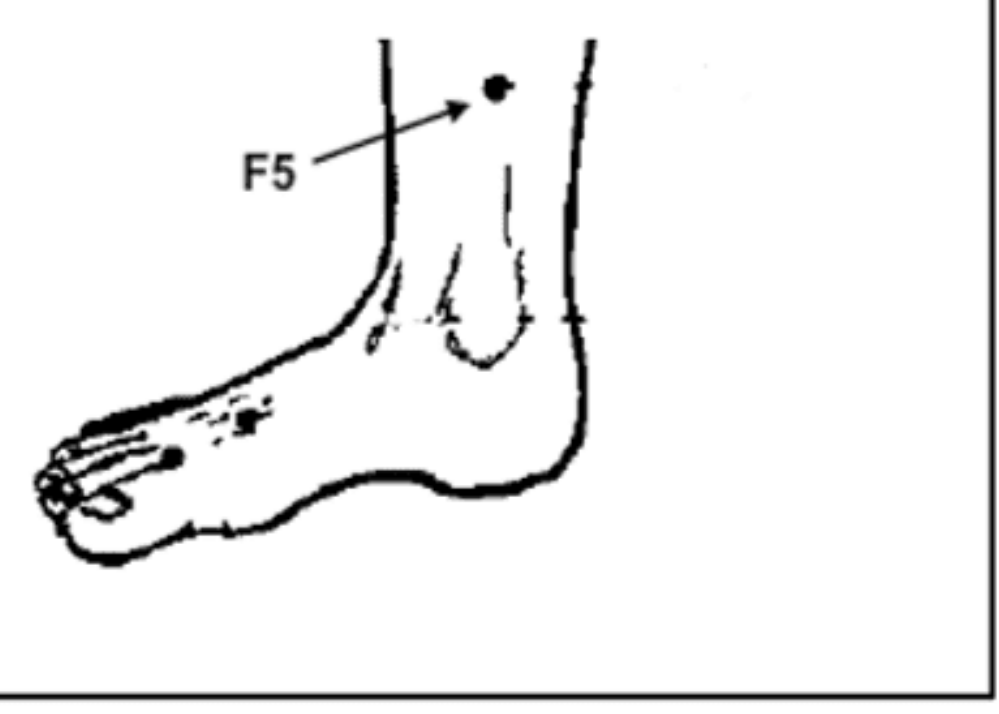

Fuente: Wen ${ }^{17}$

El estudio de $\mathrm{Akimot}^{\circ} 38$ corrobora los resultados de varios estudios relacionados con la mejora de la capacidad física y mental, donde probó veintiún atletas de fútbol durante la fase de competición, evaluando bioquímicamente la IgA salival y el cortisol, por tasa tensión muscular y fatiga, lo que resulta en un aumento del cortisol y menor IgA salival, lo que confirma la mejora efectiva en el rendimiento atlético y mental.

En otra investigación realizada en el tratamiento de lesiones deportivas con acupuntura cinética (asociación de acupuntura con kinesioterapia), en atletas de tenis, fútbol, voleibol, gimnasia olímpica, capoeira, fardo y jiu-jitsu; pacientes con trastornos locomotores tales como: tendinitis de Aquiles, dolor de espalda baja, dolor de espalda, prarcicolo, tenosinovitis, síndrome del túnel carpiano, síndrome de pronador, contusión muscular, codo de tenista y gonalgia debido a una lesión de menisco. Los 31 atletas probados regresaron al entrenamiento, como máximo, hasta la tercera sesión: $53 \%$ regresó después de la primera sesión; 37\% después del segundo; $11 \%$ después de la tercera, concluyendo que esta técnica actuó eficientemente acelerando 
el tiempo de recuperación de los atletas. La explicación de esto es que la acupuntura actúa en la inhibición del ciclo del espasmo - dolor, dando lugar a un bloqueo aferente segmental, bloque descendente supraespinal, a través de vías piramidales y la activación del proceso analgésicos endógenos. ${ }^{26}$

En otro estudio, las variables de máxima resistencia, resistencia explosiva, resistencia anaeróbica y velocidad en corredores velocistas de alto rendimiento se evaluaron durante un período de transición. El meridiano hepático se centró: hígado 1; hígado 3; hígado 8 (Figura 11A), porque las funciones energéticas de este órgano son almacenar sangre, controlar la dispersión, drenaje y determinar las condiciones del tendón y los ligamentos, además de controlar la parte emocional, porque un déficit de energía puede desencadenar: depresión; irritabilidad; insomnio; sueños perturbadores. El primer acupunto perforado fue la vejiga 62, la vesícula biliar 34, el riñón 3 (Figura 11B), seguido del bazo del páncreas 3 (Figura 1A), el hígado 1, el hígado 3, el hígado 8 (Figura 11A), el estómago 36 (Figura 1B) y el cierre con el intestino delgado 3 (Figura 3B). Los puntos de hígado se introdujeron en la octava sesión. Los resultados fueron positivamente impactantes, desarrollando una mejora en las funciones estudiadas. ${ }^{24}$ 
Figura 11 A: hígado 1 - 0,1 tsun por encima del ángulo lateral del lecho de las uñas del dedo del pie y el hígado 3 - entre el primer y el segundo metatarsal, detrás de las articulaciones metatarsal-falangeal; hígado 8 - al final del lado medial del pliegue popliteal, en el borde antero-medial de los músculos semimembranos y semitendinosos. B: riñón 3 - entre el borde posterior del maléolus medial y el tendón de Aquiles; vesícula biliar 34 - I tsun por debajo de la rodilla, en la depresión anterior e inferior de la cabeza del perobula, en la fascia del músculo peroreano largo; bexia 62 - 0.5 tsun por debajo del maleol externo, en la depresión inferior del maleol.

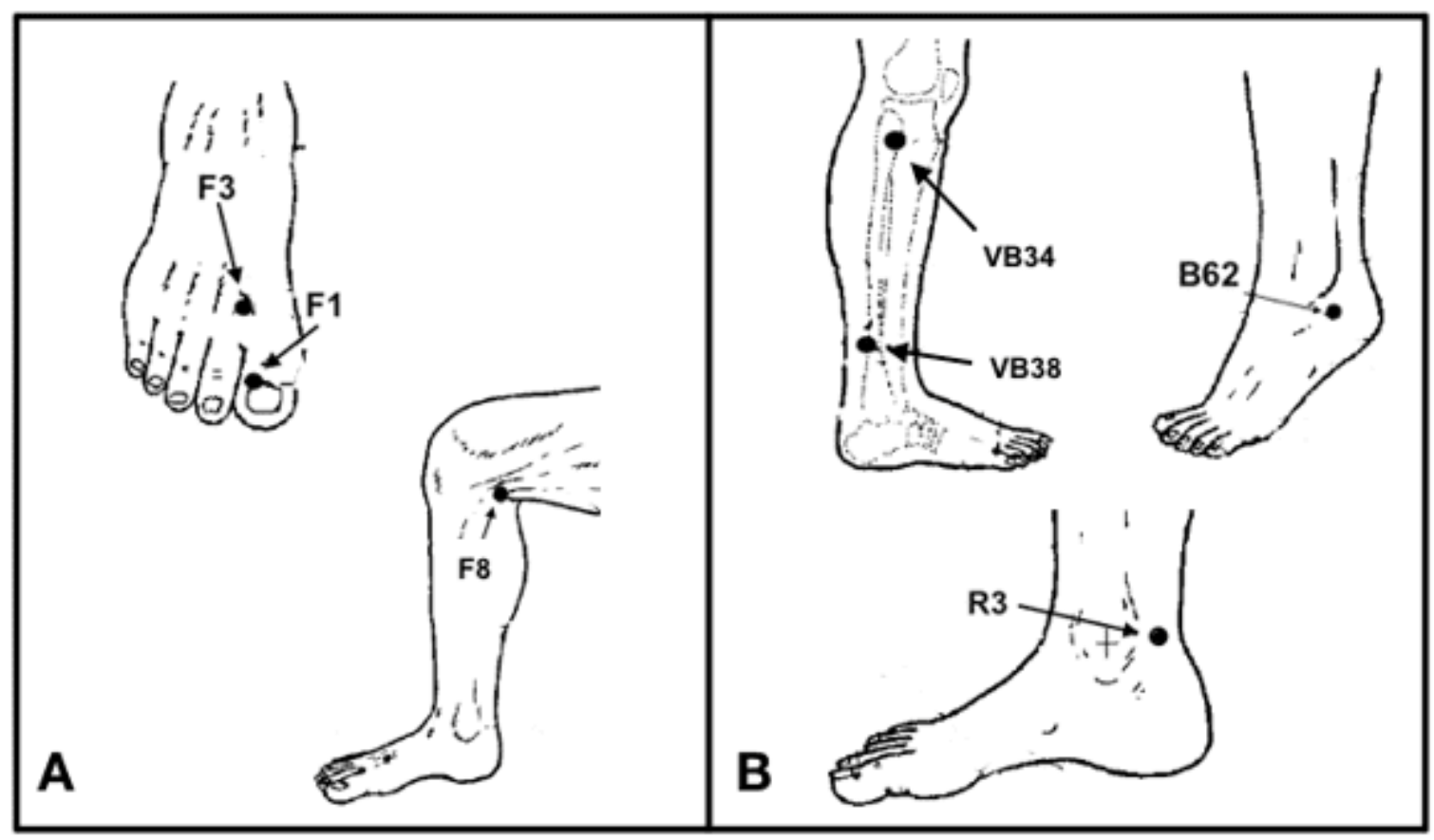

Fuente: Wen ${ }^{17}$

Ehrlich ${ }^{25}$, por otro lado, dividió 36 individuos masculinos en tres grupos: uno tratado con acupuntura sistémica; otro grupo de control; otro con acupuntura placebo. Se obtuvo una mejora significativa en el rendimiento físico y los parámetros hemodinámicos. Se llevó a cabo una sesión por semana en la vaso 20 del gubernatura, la concepción vaso 15 y el hígado 13, la vejiga 43 (Figura 12), el estómago 36 (Figura 1B) y el páncreas del bazo 6 (Figura 1A) durante 5 semanas. Analizaron que el umbral anaeróbico mejoró un 6,62\% y el rendimiento máximo en el 
$7,15 \%$ en el grupo que recibió acupuntura sistémica, ya en el grupo de control y placebo, no hubo alteraciones.

Figura 12: jarrón regulador 20 - en la línea central-vertical; 7 tun por encima del borde posterior del cabello; 5 tun detrás del margen anterior del cabello; vaso concepción 15 - 3 tsun por encima de Zhongwan (RM12), en la línea central del abdomen y el hígado 13 - en el borde inferior del punto final de la undécima costilla, en el lado del abdomen; vejiga 43 - 3 tsun, lateral del eje (Du-Mai), en el nivel inferior del borde inferior de la columna vertebral de la vértebra (T5).

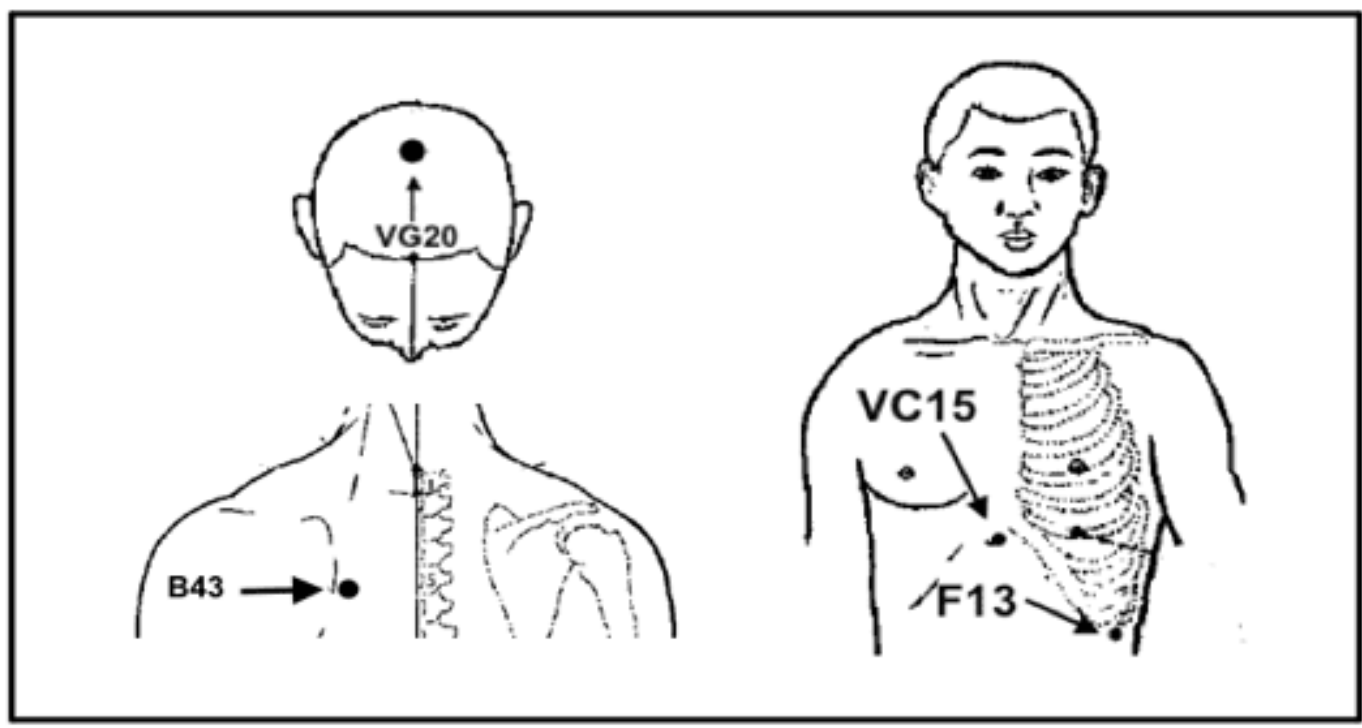

Fuente: Wen ${ }^{17}$

Lin ${ }^{40}$, evaluó los efectos de la acupuntura del oído en la frecuencia cardíaca (Cmf), el consumo de oxígeno (VO2max) y el ácido láctico en sangre en atletas de baloncesto de élite. Se dividieron en 2 grupos de 12 atletas cada uno, donde: Grupo 1 acupuntura auricular; Grupo 2 - control. Cada uno de los atletas pedaleó en bicicleta de ejercicio hasta el agotamiento, y la Cmax, VO2max y ácido láctico en sangre se midieron durante el período de descanso, después del calentamiento y durante el ejercicio: después de 5, 30 y 60 minutos. Los resultados mostraron que la $\mathrm{HR}$ y el ácido láctico en sangre fueron significativamente más bajos que en el grupo de control, después de 30 y 60 minutos de actividad. 
El mismo autor, Lin ${ }^{41}$, en una nueva investigación, evaluó las mismas variables que el FmaxC, VO2max y el ácido láctico en sangre en 30 atletas de baloncesto, divididos en 3 grupos: 1- acupuntura sistémica; 2- grupo falso; 3-control. En el grupo 1, se utilizó el punto de circulación y sexualidad 6 (Figura 13), estómago 36 (Figura 1B) y durante el estudio cada atleta realizó las pruebas en una cinta de correr de ejercicio durante 15 minutos. Las variables se midieron en reposo y en 5, 30 y 60 minutos después del ejercicio, obteniendo una reducción significativa de las tres variables después de 30 y 60 minutos.

Figura 13: circulación y sexualidad 6 - 1 tsun por debajo del punto jianshi (PC5); 2 tsun por encima de la muñeca, entre tendones de músculos palmar largos y flexor carpáculo-radial

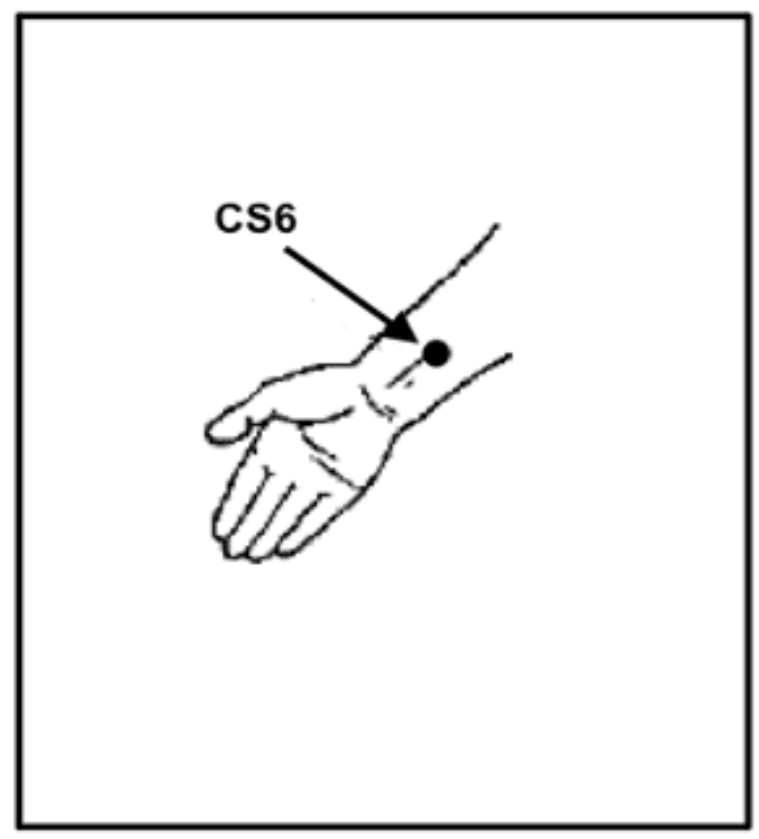

Fuente: Wen ${ }^{17}$

Dhillon ${ }^{42}$, evaluó el rendimiento de 20 ciclistas masculinos, subdivididos en tres grupos: tratados con acupuntura sistémica: estómago 36 (Figura 1B), vesícula biliar 34 (Figura 11B), hígado 11 (Figura 14), riñón 3 (Figura 11B), vaso regulador 20 (Figura 12 ); acupuntura falsa; control, sin intervención. Los atletas pedalearon en bicicleta estática durante $20 \mathrm{~km}$ a su máxima velocidad de apoyo. La escala analógica del dolor, 
visual, para las extremidades inferiores, inducida por el ejercicio; La escala de esfuerzo percibido de Borg; concentraciones de lactato sanguíneo después del ejercicio. El grupo inducido por la acupuntura presentó una puntuación más alta en la Escala Borg, lo que significa que el tratamiento promovió un mayor esfuerzo durante la actividad y disminuyó el tiempo de finalización de la prueba.

Figura 14: hígado 11 - 1 tsun por debajo de ing-inal; en el borde antero-medial del umbral del músculo aductor largo

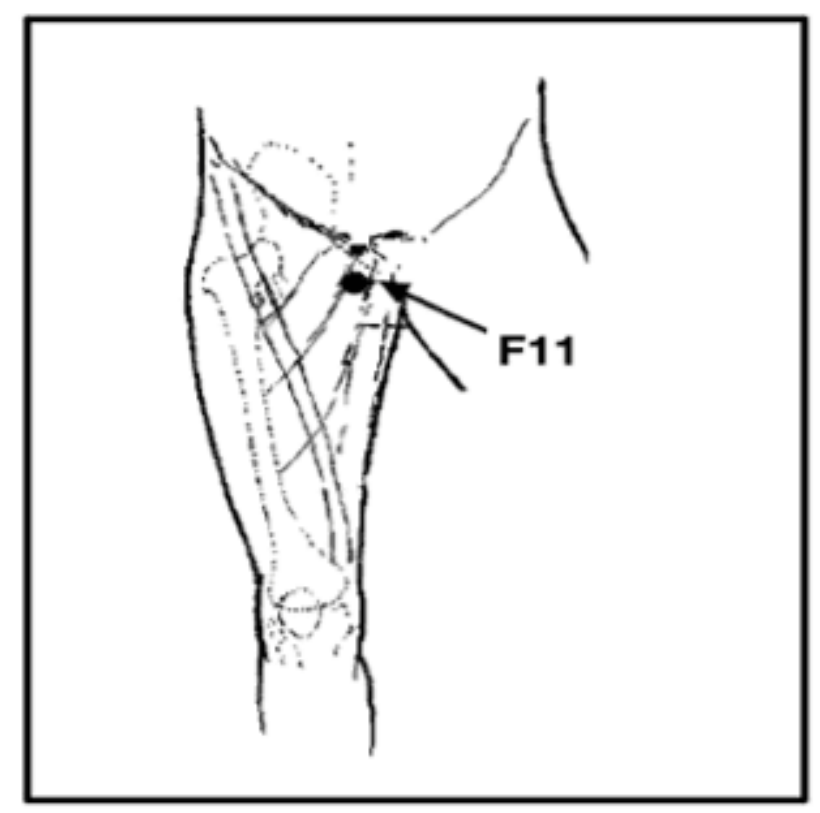

Fuente: Wen ${ }^{17}$

Según Hubscher ${ }^{43}$, evaluó el salto máximo con aterrizaje bipodal y la fuerza isométrica máxima voluntaria de los cuádriceps, con electromiografía superficial para medir la actividad muscular del femoral recto durante 30 segundos de apoyo. Treinta y tres atletas fueron divididos (13 mujeres y 20 hombres) en tres grupos: acupuntura sistémica usando puntos de estómago 36 (Figura 1B), páncreas de bazo 6 (Figura $1 A$ ), vaso concepción 6 (Figura 9); grupo de acupuntura con puntos inexistentes; Grupo de control utilizado láser desactivado. Como resultado encontrado, fue un aumento no significativo en el salto máximo, pero un aumento importante en la fuerza isométrica de los cuádriceps. 
En otra encuesta de Geng ${ }^{44}$, reclutó a 12 hombres sanos con estudiantes de educación física entre 19 y 25 años de edad, evaluando los efectos de la acupuntura del oído en los niveles de ácido láctico en sangre, inducido por el ejercicio en la cinta de correr $\left(\mathrm{V}^{\circ} 2 \mathrm{max}\right)$. Eligió los puntos: Hígado, Pulmón, Subcortex, Glándulas endocrinas y Triple Calentador (Figura 15). Los resultados fueron un aumento en la capacidad de oxígeno y disminución de los niveles de ácido láctico en sangre después de los ejercicios.

Figura 15: Puntos de oído

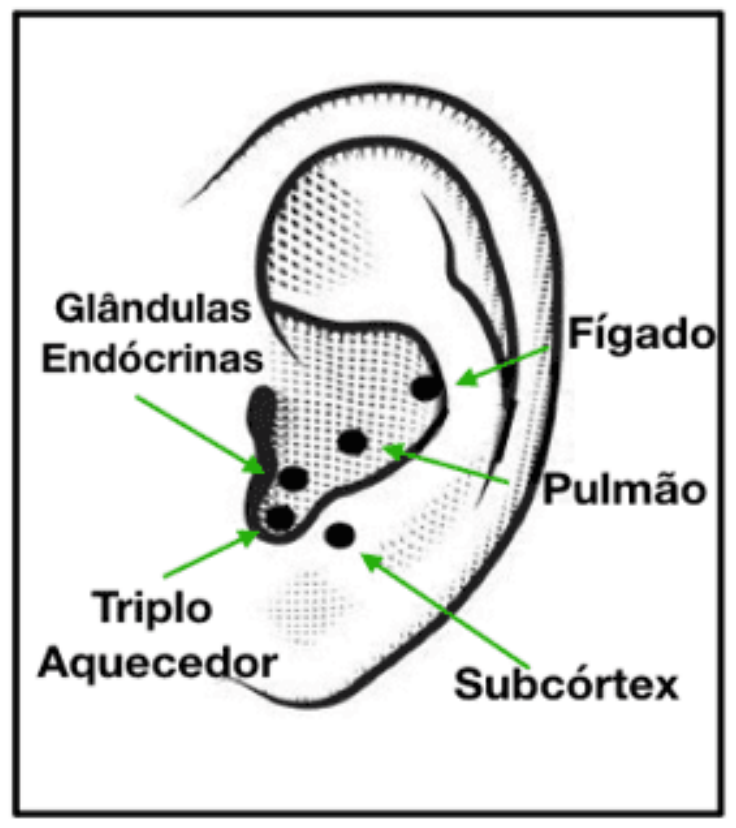

Fuente: Archivo Personal del Autor

Otro estudio de 28 individuos masculinos divididos en un grupo de control y experimental. Se realizó una recopilación de datos antes de las sesiones de acupuntura: Medición de la frecuencia cardíaca basal, a través del oxímetro; medición de la frecuencia cardíaca después de las pruebas físicas en el Banco de Harvard, con una altura de $50 \mathrm{~cm}$, realizando ascensos y descensos durante 5 minutos; frecuencia cardíaca medida en 1 minuto y medio de la prueba, 2 minutos y medio y 3 minutos y medio; cálculo del índice de aptitud física (IAF); frecuencia cardíaca promedio y el número de ascensos y descensos durante las pruebas del banco de Harvard. Los 
puntos se utilizaron: pulmón 10 y vaso concepción 17 (Figura 16), intestino delgado 3 (Figura 3B), con el fin de tonificar y conducir a la relajación muscular, fortalecimiento de tendones, columna lumbar y extremidades inferiores. En los puntos 34 de la vesícula biliar (Figura 11B), el páncreas del bazo 4 y el riñón 7 (Figura 16) se relacionaban con la sedación para potenciar el movimiento de la circulación sanguínea (Xue) y la linfa (Jing Yie). Se realizaron ocho sesiones, una cada semana y los resultados no mostraron una mejora significativa en relación con la frecuencia cardíaca y la aptitud física. Al aumentar el rendimiento físico, las subidas y descensos del Banco de Harvard, los valores fueron significativos para el grupo experimental en relación con el control. ${ }^{36}$

Figura 16: pulmón 10 - en el lado palmar, por encima de la articulación del primer metacarpiano digital, entre pieles oscuras y claras; concepción del jarrón 17 - en la línea mediana del esternón a nivel del pezón; riñón 7 - 2 tsun por encima de Taixi (R3); en el borde antero-medial del músculo de la suela; páncreas de bazo 4 - en el lado medial del pie; 1 tsol detrás de la articulación metatarsophal, en la unión de piel oscura y clara.


Fuente: Wen ${ }^{17}$ 
Fonseca ${ }^{45}$, por otro lado, utilizó una muestra con 40 individuos masculinos que practican la tensión muscular en dos grupos: control e intervención para evaluar los efectos de la acupuntura en la resistencia muscular localizada (LRE) de las extremidades superiores. El grupo de control se sometió a la prueba RML durante 1 minuto, pasando por un período de descanso de 30 minutos y después, la prueba se repitió.

En el grupo de intervención, se aplicó la prueba: la prueba; descansó durante 5 minutos; aplicación de la acupuntura, toniendo los puntos: páncreas del bazo 3 (Figura 1A); riñón 7 (Figura 16); estómago 36 (Figura 1B); jarrón del gobernador 4; triple calentador 15 (Figura 7); durante 25 minutos. Después de la aplicabilidad, la prueba de $L R L$ repitió, resultando en una mejora significativa en el rendimiento muscular del grupo de intervención.

En 2015, Moniz, utilizó 12 atletas de Rugby donde se distribuyeron en grupo de control y grupo de prueba, realizando acupuntura durante 20 minutos en los puntos: corazón 5, vejiga 15, vejiga 44 (Figura 17) y circulación y sexualidad 6 (Figura 13). En la segunda sesión semanal, se realizaron pinchazos en los puntos de tensión y los puntos de activación. Los resultados sugirieron claramente que la aplicación de la acupuntura trae ventajas a los atletas sobre los problemas musculares, con la recuperación total a nivel de dolor y la limitación de los movimientos antes de la posterior jugada de la lesión. 
Figura 17: corazón 5 - en el lado ventral y cubital del antebrazo, en el lado cubital del tendón del músculo flexor cubital carpiano; 1 tsun por encima de la manija; vejiga 15 - en el nivel del borde inferior del proceso espinal de la vértebra (T5), en la misma línea vertical que el punto Feishu (B13); vejiga 44 - 3 tsun, lateral del eje, en el nivel inferior del borde inferior de la columna vertebral de la vértebra (T6).

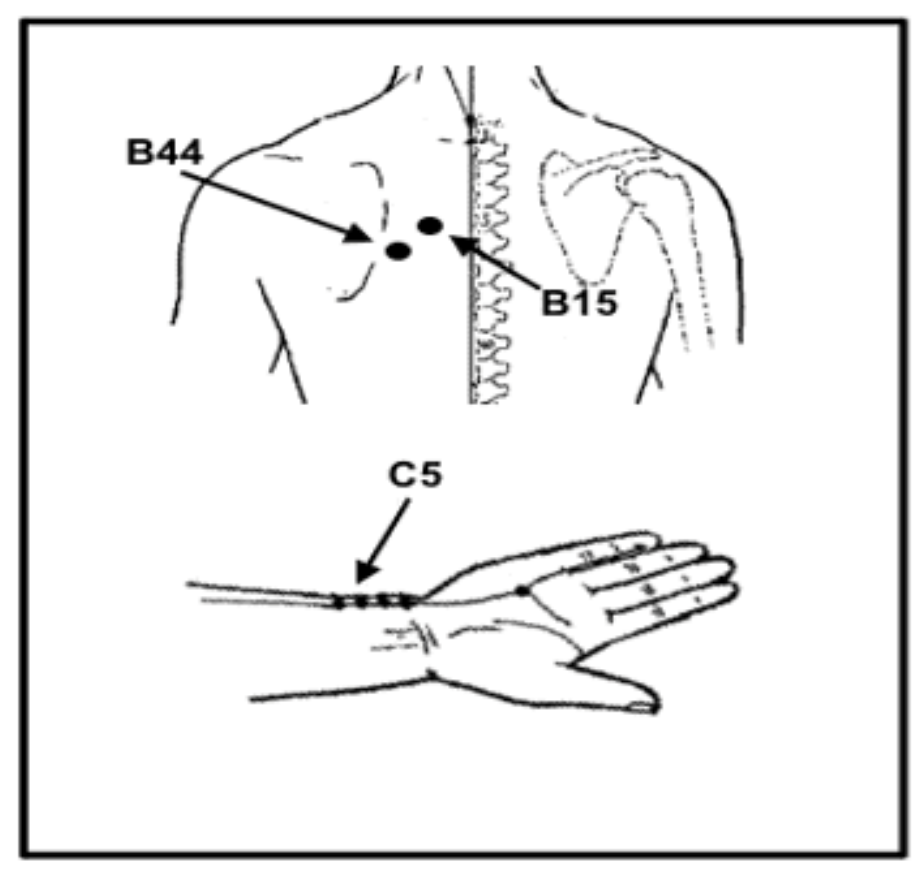

Fuente: Wen ${ }^{17}$

La mayoría de los estudios presentan resultados significativos con respecto a la mejora del rendimiento físico, lo que conduce a una mejora en el tiempo de finalización de las pruebas. Esto hace que las personas sometidas al tratamiento de acupuntura, recibiendo una activación de la neurofisiología del control del dolor, desencadenando una mejora en el rendimiento muscular, influyendo en gran medida en el resultado final. ${ }^{46}$

\section{ACUPUNCTURE UTILIZADO EN EL TRATAMIENTO DE LESIONES}

La acupuntura actúa en este proceso de curación de la enfermedad, eliminando su causa, y por lo tanto se está administrando tanto para el tratamiento de diversos 
dolores agudos como crónicos. Se puede utilizar en procedimientos quirúrgicos, en la mejora de las condiciones físicas y en la prevención de lesiones en diversos deportes. 24

Actualmente los atletas y sus entrenadores buscan la mejora del rendimiento físico y la prevención a través de un entrenamiento exhaustivo, monitoreo nutricional, prevención fisiotermapeupica, planes de entrenamiento, monitoreo psicológico y uso de nuevos equipos deportivos de consecución de objetivos. Incluso con estos sistemas mejorados, muchos atletas prefieren y se mueven por el camino más rápido, utilizando tratamiento ilícito, como el uso de anabólicos y drogas. ${ }^{32}$ Según Frasca 35, afirma que la acupuntura funciona en el ser humano en su conjunto, tratando el dolor, mejorando el rendimiento y actuando directamente en el sistema psicológico de los atletas generando un aumento en la confianza psíquica del deportista.

Según Santos y los colaboradores ${ }^{20}$, la mayoría de los atletas han tenido la experiencia de sentir dolor muscular de inicio tardío, que aparecen en los períodos de entrenamiento, donde la intensidad y el volumen de trabajo son más altos. Otro tipo de dolor es causado por una lesión, alejando a los atletas del entrenamiento y las competiciones durante largos períodos. ${ }^{21}$

Basándose en la neurofisiología, histoanatómica y neuroquímica, CTM explica que al puntuar el punto que se encuentra en los canales de los meridianos específicos, facilitará el flujo de energía en estas estructuras sobrecargadas por modalidades deportivas, alcanzar objetivos analgésicos. ${ }^{25,47}$

Otros autores informan que la aplicación de la técnica tiene la acción de opioides endógenos, la liberación de la endorfina que se identifica en la modulación del dolor y la inhibición de la transmisión nociceptiva en todos los niveles del sistema nervioso se produce.) a través del uso de electroacucupación, se produce una regulación positiva de la expresión de la relación de síntesis de óxido nítrico neural (nNOS) / DIAphora NADPH (NADPHd). También hay algunos datos que sugieren que, además de los opioides, otros mecanismos están involucrados en el tratamiento del dolor. $6,48,49,50$, 51 
La presencia de NO favorece la mejora de la vascularización local, indicando una acción homeostótica de la acupuntura, pero el aumento del NO en el fascículo helado puede aclarar esta acción reguladora de los reflejos somáticos causados por la técnica. 6, 52 Otros estudios observan la liberación de serotonina después de la estimulación de la aguja. $51,52,53$

La acupuntura se aplica al tono, relaja los músculos de los atletas y mantiene el equilibrio energético del cuerpo. Además del efecto analgésico, es posible estimular otros factores biológicos, activando el hipotálamo y la glándula pituitaria alcanzando efectos sistémicos, tales como: aumento de las neuro-hormonas y neurotransmisores.2 mejorar el rendimiento del atleta en sus respectivas modalidades, así controlado por sistemas, nervioso, endocrino e inmune. ${ }^{36,54,55,56,57}$, 58, 59, 60 Muchos estudios han demostrado que la acupuntura funciona en estos tres sistemas, proporcionando: alivio de las tensiones y dolor muscular; aumento del flujo sanguíneo local; liberación de opioides endógenos, disminuyendo la percepción del dolor; mejora de la modulación del equilibrio del sistema nervioso simpático y parasimpático; modulación del sistema inmunitario; activación del tejido conectivo y los músculos liberando miosinas que tienen efectos antiinflamatorios, liberación de sustancias antioxidantes, disminución del estrés oxidativo; sustancias que tienen efectos ergogénicos probables. $5,13,61,62,63,64$

En un estudio de laboratorio con ratas, Lee y colaboradores, 65 informaron que el animal cuando se sometió a la acupuntura y ejercicio presentó una supresión de los efectos en 5 HT (5 hidroxitriptiminas) y la expresión de THP (hidroxilasa triptófano) en el balsofa dorsal de conejillos de indias, alcanzando el efecto ergogénico, ya que el 5 HT impide la realización de ejercicio en humanos y ratas.

En un estudio, Zyloney y 66 colaboradores informaron que es posible encontrar una actividad diferente en el cerebro, a través de la evaluación de resonancia magnética, durante el procedimiento de la aguja. Estos cambios aparecen en regiones tales como: materia gris epiaquedural, hipotálamo, corteza somatosensorial primaria, gisrus temporal superior, corteza cingulada anterior rostral y región medial de la corteza occipital. 48 
En su investigación, Mino 67 trató lesiones repetitivas de estrés del hombro, con la combinación de maravillosas puntadas de vaso: triple calentador 5 - vesícula biliar 41 (Figura 18) que se utilizan para el dolor crónico y el intestino delgado 3 (Figura 3B) vejiga 62 ( Figura 11B) para el dolor agudo.

Figura 18: triple calentador 5 - 2 tsun por encima del pliegue dorsal de la muñeca, entre los tendones del músculo extensor digital común y el músculo de quinto dígito en sí; vesícula biliar 41 - en depresión entre el cuarto y quinto metatarsal.

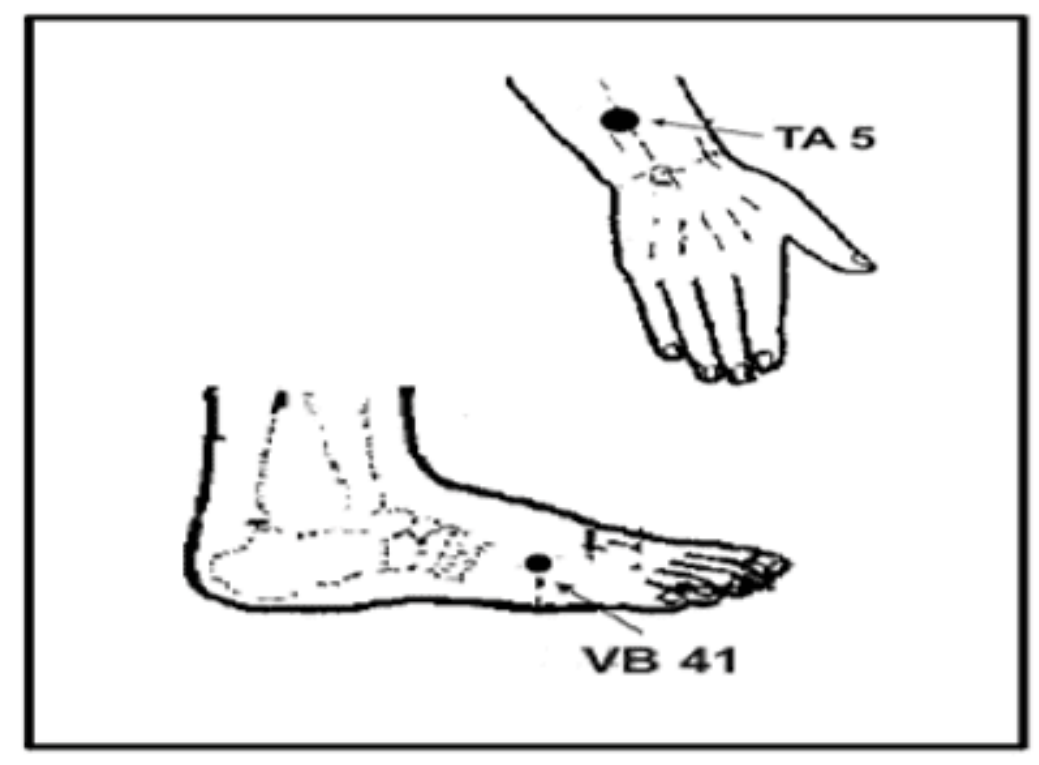

Fuente: Wen ${ }^{17}$

Los puntos locales para el tratamiento son: intestino grueso 5 para el dolor de muñeca; intestino grueso 4 alivia el dolor y los edemas (Figura 4 A); intestino delgado 9 dolor en el brazo; triple calentador 14 indicado para el dolor y la inflamación del hombro; intestino grueso 14 (Figura 19) utilizado para la rigidez, paresia, movilidad reducida del hombro. Puntos distantes, dolor de hombro superior y anterior: estómago 36 para disipar factores patógenos externos; estómago 37 indicado para el dolor, sensibilidad y trastornos de la circulación; el estómago 41 elimina la humedad, el estancamiento, el viento y alivia la carga (mente); el estómago 38 elimina el dolor agudo y los trastornos articulares del hombro (Figura 1B). Para el dolor de hombro posterior y superior: vesícula biliar 34 indicado para movilidad reducida, espasmos, trastornos de 
circulación y trastornos funcionales de los músculos y tendones; la vesícula biliar 38 dispersa el viento y libera calor, activa el meridiano y sus vasos (Figura 11B). La escápula del dolor, por otro lado: la vejiga 65 pacifis y fortalece el espíritu, libera calor y alivia el dolor; la vejiga 64 suprime los espasmos y relaja los tendones; la vejiga 60 alivia el dolor y activa los puntos meridianos y emocionales (Figura 2A); La vejiga 18 promueve la calma general y elimina los espasmos (Figura 19) y el hígado 8 relaja los tendones (Figura 11A).

Figura 19: intestino delgado 9 - en la región posttero-inferior de la articulación del hombro, en el lado postteroinferior del músculo redondo más grande; calentador triple 14 - en depresión entre el ahumórico y el húmero tuberculo más grande, en el borde del tendón infraespinal; intestino grueso 14 - cubre el lado y algún radial del brazo, en el punto distal del músculo deltoides, 3 tsun por debajo del punto jianyu; vejiga 18 - en la misma línea vertical que geshu (B17), en el nivel inferior del proceso espinal de la vértebra $(\mathrm{T} 9)$.

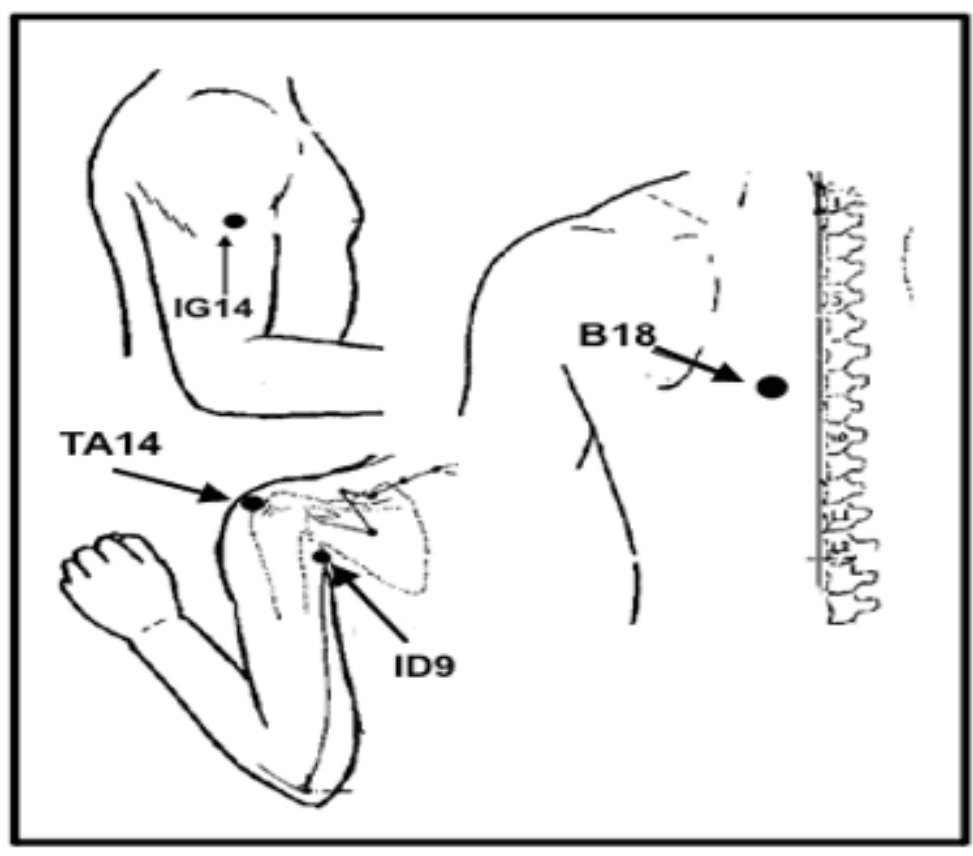

Fuente: Wen ${ }^{17}$ 
Brum ${ }^{68}$, por otro lado, asociado en su estudio para tratar el síndrome piriforme, técnicas de masaje y acupuntura en los pasillos, con el fin de mejorar las funciones musculares, flexibilidad y alivio de los síntomas. La investigación incluyó atletas de ambos sexos de 30 a 60 años. La sesión se centró en el masaje (Shiatsu) al principio para liberar los puntos de tensión de la región glútea y muslo en el lado afectado, seguido de agujas, de la siguiente manera: en el sentido de las agujas de las agujas; anti-tiempo; profundización; superficialización de la aguja en las siguientes regiones: origen e inserción del músculo piriforo (cara pélvica del sacro y trocanter más grande del fémur); origen del glúteo medio (cara lateral del hueso ilíaco); límite superior del glúteo mínimo; límite superior del glúteo máximo; origen de los músculos isquiotibiales (tuberosidad ciática); bíceps femoral muscular y diafísica muscular semitendinosa, terminado con masaje.

Los resultados mostraron una mejora significativa, cuando se relaciona con la mejora del dolor, flexibilidad, palpación del dolor en el músculo piriformo y prueba para el síndrome piriforme. Esto muestra el acuerdo con el estudio de Hongwen 69, donde la eficacia de la acupuntura fue del 97,33\% y con la investigación de Francia 26 que asoció las agujas con la kinesioterapia alcanzando un alivio de los síntomas, la potencialización del sistema neuromuscular, proporcionar un retorno más rápido a las actividades deportivas.

En su trabajo, Rocha ${ }^{70}$, verificó la mejora de la activación del músculo femoral del recto, a través de la electromiografía, después de la estimulación de la acupuntura estomacal punto 45 (Figura 20). Un total de 10 participantes ( 5 machos y 5 hembras) fueron seleccionados, donde realizaron un ejercicio de 2 repeticiones en cuclillas, donde seleccionaron el índice de activación muscular más alto entre los dos movimientos por la señal electromiográfica. Luego se aplicó la acupuntura, tonificación del punto del estómago 45 durante 10 minutos y luego se repitió la sentadilla isométrica en la fase inferior. Según los resultados encontrados la acupuntura alteró la actividad eléctrica del músculo femoral del recto durante la fase isométrica en la fase inferior de la sentadilla, demostrando ser eficaz. 
Figura 20: estómago 45 - 0.1 tsun en el lado de la esquina lateral del lecho de uñas del segundo dedo del pie

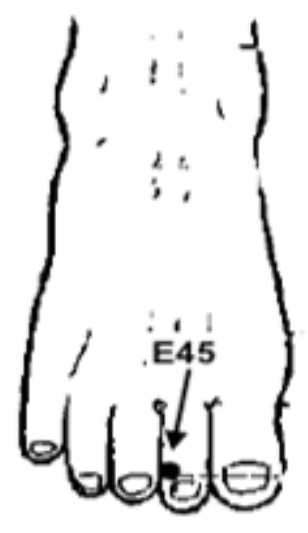

Fuente: Wen ${ }^{17}$

En otro estudio Pinheiro ${ }^{71}$,caracterizado y presentó algunos puntos para el tratamiento de la lesión meniscal: hígado 8 (Figura 11A) utilizado para tonificar el canal hepático con función de armonización y tonificar el hígado y la sangre Qi, promoviendo la plenitud de los riñones y lo que conduce a la relajación de tendones y músculos, fortaleciendo el coeficiente intelectual de la rodilla. El punto de vejiga 40 (Figura 5) se encuentra en la fosa popliteal que permite: dispersión del calor y viento perverso de las extremidades; relajación de los músculos y tendones; destruir los vasos sanguíneos; reduce el calor y elimina la éxtasis de la sangre; disminución de la regidez y fortalece la rodilla y la lumbar. Por otro lado, el punto renal 10 (Figura 21) moviliza el agua; tonos yin Qi; expulsa la humedad y el calor perverso, el tratamiento del dolor de rodilla, el edema de las piernas y el entumecimiento de la rodilla y el cessum popliteal. 
Figura 21: riñón 10 - en el lado medial del pliegue popliteal, entre los músculos semitendinosos y semimembranos

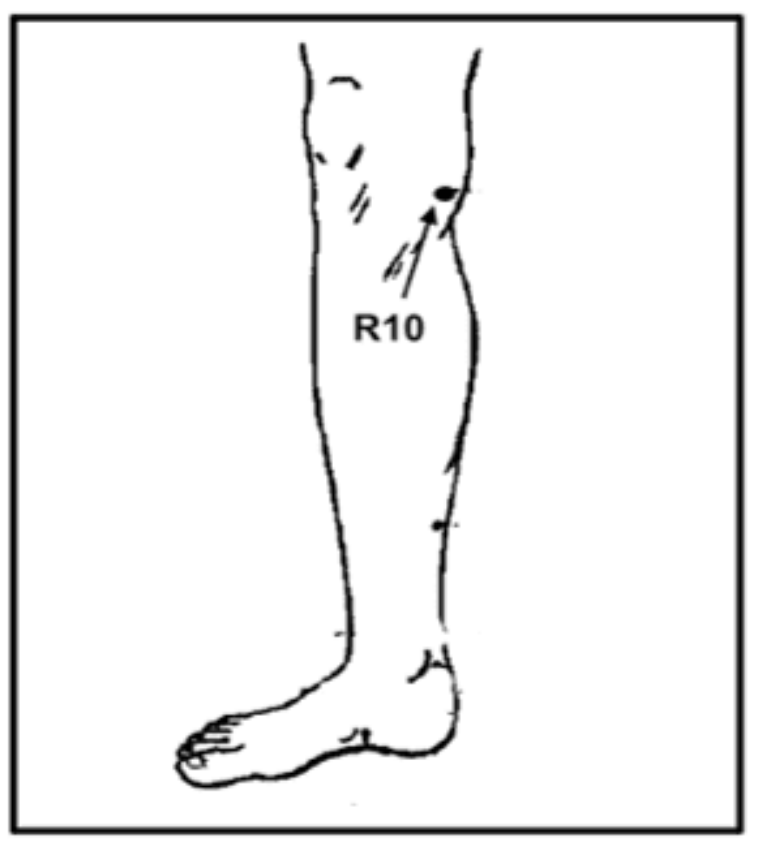

Fuente: Wen ${ }^{17}$

En un estudio sobre las disfunciones del hombro, Nunes ${ }^{72}$, revisó la bibliografía y archivó los puntos de los vasos maravillosos: intestino delgado 3 (Figura 3B) - vejiga 62 (Figura 11B) para equilibrar las funciones, músculos relajantes y tendones; los puntos del triple calentador 5 - vesícula biliar 41 (Figura 18) aliviar el dolor y relajar los tendones; Puntos Ashi; hígado 8 (Figura 11A) y riñón 7 (Figura 16) para fortalecer los tendones; estómago 36 (Figura 1B) eliminando el frío y la humedad de las articulaciones; intestino 4 (Figura 4A) e intestino grueso 15 (Figura 22) actuando en el movimiento y dolor de la flexión del hombro; triple calentador 14 (Figura 19) e intestino grueso 16 (Figura 22) mejorando la movilidad de extensión y el dolor. 
Figura 22: intestino grueso 15 - en la parte superior del hombro, en el borde lateral del acromion, hay dos depresiones, este punto está en la depresión anterior; intestino grueso 16 - en la depresión entre el borde superior e posterior de la articulación acromioclavicular y la columna vertebral de la escápula.

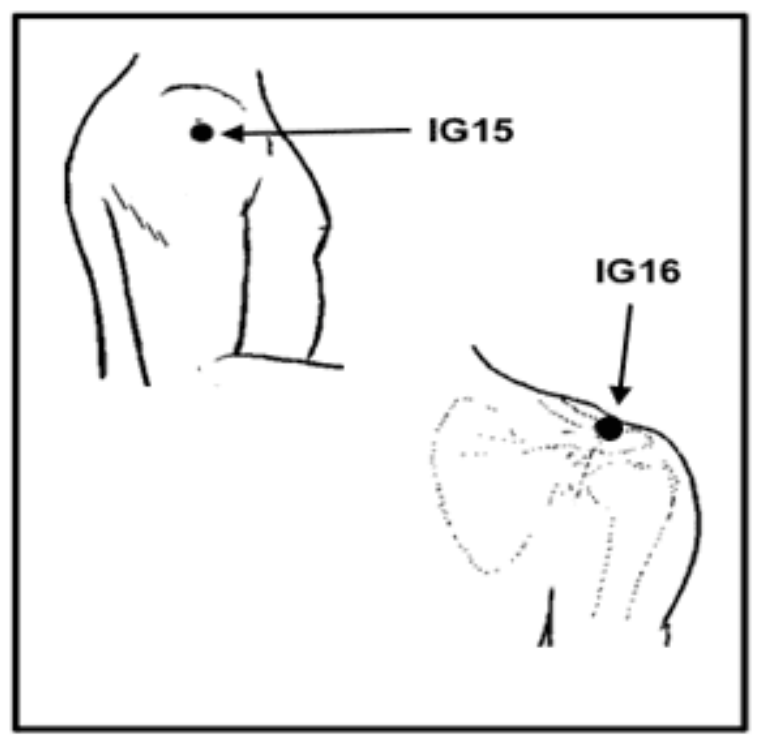

Fuente: Wen ${ }^{1}$

Con el fin de verificar los efectos inmediatos de la acupuntura en el dolor muscular de inicio tardío (DMIT) y la capacidad funcional contráctea de los músculos, Parisoto 73 , seleccionado 30 sujetos (12 hombres y 18 mujeres), con una edad de entre 20 y 30 años, practicantes de actividad 2 a 3 veces por semana. Se dividieron en tres grupos: tratados con acupuntura; farsa placebo; Control. Se evaluaron los siguientes: umbral de dolor con el algelometer; máxima fuerza muscular isométrica máxima; actividad electromiográfica; percepción subjetiva del dolor a través de la escala analógica visual.

Después de las evaluaciones, los voluntarios fueron sometidos a inducción de DMIT, realizando flexión y extensión del codo con carga (hilo de bíceps), hasta el límite máximo de ejecución del movimiento: 3 series se realizaron hasta el agotamiento muscular con un intervalo de 4 a 5 minutos. Después de este procedimiento, se reevaluó el estado en el que estaba el músculo antes de la acupuntura. 
Los puntos elegidos para el tratamiento del grupo de acupuntura fueron: intestino grueso 4 (Figura 4A), intestino grueso 11 (Figura 4B), estómago 36 (Figura 1B), vesícula biliar 34 (Figura 11B), que son analgésicos y puntos tonantes de los músculos $\mathrm{y}$ tendones. En el grupo falso, se utilizaron puntos placebo y las agujas se introdujeron superficialmente; grupo de control de los individuos acaba de establecer. Ambos grupos permanecieron en reposo durante 20 minutos.

Los resultados indicados: la percepción del dolor con la escala analógica se redujo en el grupo de acupuntura; el umbral de dolor por el algelometer aumentó después de la intervención en este grupo. Después de la intervención de acupuntura, la activación muscular del bíceps se redujo en un 30\% cuando está relacionada con la farsa y el grupo de control. La fuerza muscular, después del DMIT, disminuyó en un $48 \%$ en el grupo probado, siendo menor que en los grupos de farsa y control (58\% y 56\% respectivamente). Todos los grupos presentaron el déficit máximo de fuerza muscular isométrica después del protocolo de inducción dmit: la acupuntura disminuyó en un $26 \%$; una farsa del $29 \%$; control $33,5 \%$.

Sin embargo, se concluyó que la acupuntura era eficaz en la mejora inmediata de la percepción del dolor y el aumento del umbral por algelomer, proporcionando alteraciones neuromusculares, pero no mejorando el rendimiento contractilo de la musculatura.

En otro estudio de Tolentin ${ }^{74}$, evaluó el efecto de la auriculoterapia en el dolor, la funcionalidad y la movilidad de las personas con dolor de espalda baja crónica. Dividido 31 participantes en tres grupos: experimental con agujas de sangre inoxidable desechables de 1,5 mm; experimental con semillas de mostaza; control sin intervención. La escala analógica visual se utilizó para evaluar el dolor; para la prueba de funcionalidad, se utilizaron el cuestionario de discapacidad lumbar de Quebec y la prueba de estar sentada; se realizó movilidad lumbar.

Las intervenciones se realizaron durante 4 semanas, siendo una sesión semanal, aplicada en los puntos: Shenmen, Rim, Sympathetic, Analgesia, Muscle Relaxation, 
Lumbar y Adrenal Spine (Figura 10). Después de un intervalo de 1 semana después de la última aplicación, los individuos fueron reevaluados con el mismo protocolo.

Los resultados mostraron una mejoría en la condición del dolor de espalda baja, así como la mejora de la capacidad funcional en ambos grupos experimentales, a diferencia del control de que no había diferencia significativa en relación con el dolor inicial. No se encontró diferencia entre las técnicas realizadas (aguja y semilla), lo que indica que ambas son beneficiosas para el alivio del dolor crónico de espalda baja y el aumento de la capacidad funcional.

Figura 23: Puntos de oído

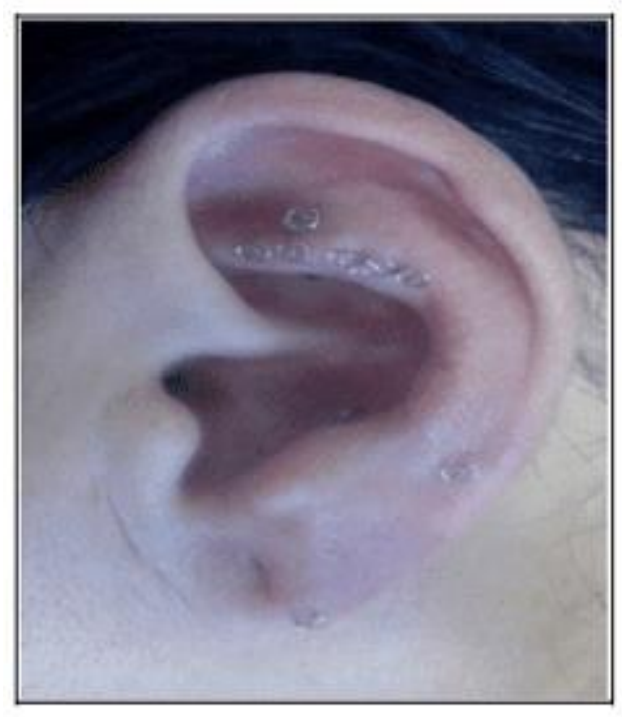

Fuente: Tolentino ${ }^{74}$

\section{CONSIDERACIONES FINALES}

Basado en la literatura, analizando todos los resultados y su evidencia científica, se considera que el tratamiento de la acupuntura es eficaz en el tratamiento, mejora del rendimiento y prevención de lesiones en atletas profesionales y aficionados. Individuos que reciben la intervención, incluso si tienen algún tipo de complicación lesionada, el problema es menos grave y restaurar rápidamente la función orgánica, 
es decir, el atleta que sufre algún tipo de lesión y está realizando acupuntura, esta adversidad tenderá a ser actividades de retorno mínimo y temprano.

Incluso los atletas que no utilizan la acupuntura para prevenir la prevención y presentar la lesión, la intervención de esta presenta evidencia clínica, basada en neurofisiología, liberación de opioides, con énfasis en el tratamiento del dolor y la mejora del bienestar del paciente.

La intervención de acupuntura, aunque es una técnica milenaria, sigue creciendo en Occidente, ha estado realizando grandes resultados en la práctica clínica, cuestiones de prevención de lesiones musculoesqueléticas, y también en la prevención y tratamiento de enfermedades dolor psicosocial, agudo y crónico.

Debido al equilibrio del sistema energético, la acupuntura no sólo trata el bienestar físico, sino también el bienestar mental, porque la base teórica de la técnica no separa el cuerpo de la mente beneficiando el estrés emocional, reduciendo la ansiedad y equilibrando la mente humana.

\section{REFERENCIAS}

1. Ching N. O livro de ouro da medicina chinesa. 3. ed. Rio de Janeiro: Objetiva; 1991.

2. Malveira CL, Souza FCS, Rocha TBX. Biomecânica do movimento isométrico de pedala antes e após aplicação de acupuntura: Um estudo preliminar. Motric. 2012 Set;8(52):630-9.

3. Sousa NR. Relação entre a reatividade de pontos de acupuntura e atividade física com afecções ortopédicas determinadas por exames de imagens em equinos. Botucatu. Tese [Doutorado em Biotecnologia Animal] Universidade Estadual Paulista; 2015.

4. Silveira NC, Martins RCC. Eficácia da acupuntura para o tratamento da fibromialgia: Uma revisão sistemática. Psicol e Saúde em Debate. 2018 Fev; 4(1):85-105. 
5. Sant' Anna FM, Rangel VM, Mota DDS. Tratamento da Tenossinovite de De Quervain por Acupuntura - Relato de Caso. Vittalle. 2018 Mar; 30(1):130-6.

6. Tsuchiya M, Sato EF, Inoue M, Asada A. Acupuncture enhances generation of nitric oxide and increases local circulation. Anesth Analg. 2007 Feb; 104(2):3017.

7. Tavares MG, Machado AP, Motta BG, Borsatto MC, Rosa AL, Xavier SP. Electro-acupuncture efficacy on pain control after mandibular third molar sugery. Brz Dent J. 2007; 18(2):158-62

8. Hutchinson AJ, Ball S, Andrews JC, Jones GG. The effectiveness of acupuncture in theating chronic non-specific low back pain: a systematic review of the literature. J Otthop Surg Res. 2012 Oct 30;7:36.

9. Moniz PJL. Avaliação da acupuntura no desempenho do rugby. Coimbra. Dissertação [Mestrado Integrado em Medicina] - Faculdade de Medicina da Universidade de Coimbra; 2015.

10. Magalhães PC. Efeito da acupuntura na prevenção da hemorragia pulmonar induzida por exercício em cavalos puro sangue inglês de corrida. Botucatu. Dissertação [Mestrado em Biotecnologia Animal] - Universidade Estadual Paulista; 2016.

11. Matias B et al. Acupuntura de equilíbrio como coadjuvante no tratamento de disfunções temporomandibulares. Ação Odonto. 2018 Jun; 1(1):65-72.

12. Franco A. Acupuntura Ajuda Atletas nas Lesões e na Performance. Jornal da Madeira 2016 agosto 2;p.25.

13. Lopes SS, Mota MPG. Efeito da acupuntura na resistência física após exercícios repetitivos de tornozelo - Estudo experimental. R bras Ci e Mov. 2018 Jul;26(1):13-21.

14. Lopes SS, Mota MPG. Influência da acupuntura no limiar de percepção dolorosa de musculatura submetida a esforço repetitivo. Br J Pain. 2018 Jul; $1(3): 207-11$

15. Cohen M, Abdala RJ, Ejnisman B, Amaro JT. Lesões Ortopédicas no futebol, Rev. Bras. Ortop 1997 Dez;32(12):940-44.

16. Dias Junior JC, Marino DM. Acupuntura na prevenção de lesões musculares em atletas de futebol profissional. Rev Fisioter S Fun. Jan-Jul:6(1):36-42. 
17. Wen TS. Acupuntura Clássica Chinesa. 15. ed. São Paulo: Cultrix, 2006.

18. Maciocia G. Os fundamentos da medicina chinesa: um texto abrangente para acupunturistas e fitoterapeutas. São Paulo: Roca; 1996.

19. Ross J. Combinações dos Pontos de Acupuntura: A Chave para o Êxito Clínico. 1 ed. São Paulo: Roca, 2003.

20. Santos VC, Kawano MM, Banja RA. Acupuntura na melhora da performance em atletas juvenis de handebol, Rev Saúde e Pesq 2008 Set-Dez;1(3):331-35.

21. Rubio K, Godoy Moreira F. A dor em corredores com fascite plantar: o uso da acupuntura, Rev Dor 2008 Jul-Set;9(3):1290-1296.

22. Pires TF, Pellegrinotti IL. Acupuntura na Performance Atlética: Estudo Exploratório. [8 8 $^{\mathrm{a}}$ Mostra Acadêmica da UNIMEP; 2010 out; 1-4; Piracicaba, Brasil].

23. Yang HY, Liu TY, Gao M. Electrical acupoint stimulation increases athletes rapid strength, Zhongguo Zhen Jiu 2006 May;26(5): 313-315.

24. Luna MP, Fernandes Filho J. Efeitos da Acupuntura na performance de Atletas velocista de alto rendimento do Rio de Janeiro. Fit e Perform J 2005 Julh/Ago;4(4):199-214.

25. Ehrlich D, Haber P. Influence of acupuncture on pysical performance capacity and Haemodynamic Parameters. J. Sports Med 1992 May;13(6): 486-91.

26. França D, Fernandes-Senna V, Cortez CM. Acupuntura cinética como efeito potencializador dos elementos moduladores do movimento no tratamento de lesões desportivas. Fisioter Bras 2004 mar-abr; 5(2):111-8.

27. Wadsworth L T. Acupuncture in sports medicine. Curr Sports Med Rep 2006 Feb; 5(1):1-3.

28. Barela J A. Estratégias de Controle em Movimentos Complexos: Ciclo Percepção - Ação no Controle Postural. Rev Paul de Educ Fís 2000; 3: 79-88.

29. Papler PG et al. Reabilitação do joelho. In: Greve J.M. A. e Amattuzzi, M.M. Medicina de reabilitação aplicada à ortopedia e traumatologia. São Paulo: Roca, 1999.

30. Gemeo LH. Ignatti C. Acupuntura como Ferramenta Auxiliar do Aumento da Performance Desportiva. In: Anais do Simpósio Internacional de Ciências 
Integradas da UNAERP; 2004 ; Guarujá, Brasil. São Paulo: Universidade de Ribeirão Preto Campus Guarujá; 2004. p. 1-9.

31. Rossetto SC. Acupuntura nos Esportes. 1 ed. São Paulo: Phorte, 2009.

32. Pelham TW, Holt LE, Stalker R. Acupuncture in human Performance. J Strength Cond Res 2001 May;15(2):266-71.

33. Myamoto T. Acupuncture treatment for muscle injury. Japa Journ of Phys Fit and Spor Med 1997; 43(2):39-41.

34. Fry AC, Kraemer KJ. Resistance exercise overtraining and overreaching: neuroendocrine responses. Sports Med 1997 Feb;23(2): 106-29.

35. Frasca L. Desempenho na Ponta da Agulha. Rev Farmac 2011 out-nov:44-5.

36. Costa V. Acupuntura Previne Lesões e ajuda a melhorar o Desempenho nas Pistas [periódico na internet]. 2013 [acesso em 22 jan 2017] Disponível em: http://blogs.oglobo.globo.com/pulso/post/acupuntura-previne-lesoes-ajudamelhorar-desempenho-nas-pistas-500798.html.

37. Luna M. Os Benefícios da Acupuntura no Esporte. 2016 [acesso em 22 jan 2017] Disponível em: http://www.ibramrp.com.br/noticia/49/os-beneficios-daacupuntura-no-esporte.

38. Akimoto T, Nakahori C, Aizawa K, Kimura F, Fukubayashi T, Kono I. Acupuncture and responses of imunoligic and endocrine markers during competition, Med Sci in Sports Exerc 2003 Ago;35(8):1296-1302.

39. Bopp-Limoge C. L’acupuncture Permet-elle D`ameliorer les Performances Sportives Stude Personnelle a Propos de 35 Sportifs de haut niveau. These Medicine 1998; 117:215.

40. Lin ZP et al. Effects of acupuncture stimulation on recovery ability for male elite basketball athletes. Amer Jour of Chin Med 2009; 37(3):471-81.

41. Lin, ZP et al. Effect of auricular acupuncture on oxygen consumption of boxing athletes. Chin Med Jour 2009; 22(13):1587-90.

42. Dhillon $S$ et al. The acute effect of acupuncture on 20-km cycling performance. Clin Jour of Spo Med 2008; 18(1):76-80. 
43. Hubscher $\mathrm{M}$ et al. Immediate effects of acupuncture on strength performance: a ramdomized, controlled crossover trial. Euro Jour of Appli Physi 2010; 110(2):353-58.

44. Geng $L \mathrm{~J}$ et al. Investigation on the effects of ear acupressure on exerciseinduced lactic acid levels and the implications for athletic training. Amer Jour of Acupu 1995; 23(4).

45. Fonseca LP, Lessa JFM. Efeito da Aplicação da Acupuntura na Resistência Muscular Localizada de Membros Superiores em Praticantes de Exercício Resistido. Brasília. Monografia [Graduação em Fisioterapia] - Centro Universitário de Brasília; 2011.

46. Belmiro H, Vicentini D, Camilotti CM. Efeitos da Acupuntura no Desempenho Motor de Atletas. Rev Fac Educ Fis 2013 jul-set; 11(3):176-91.

47. Angeli AL, Fernandes JGJ, Luna SPL. Acupuncture Applied Equine Sports Medicine, Ver Acad 2007 Jul-Set;5(3):325-33.

48. Staud R. Mechanisms of acupunture analgesia: effective therapy for musculoskeletal pain? Curr rheumatol Rep. 2007 Dec;9(6):473-81

49. Bucinskaite V, Lundeberg T, Stenfors C, Ekblom A, Dahlin L, Theodorsson E. Effects of electro-acupuncture and pysical exercice on regional concentrations of neuropeptides in rat brain, Brain research 1994 Dec;666(1): 128-32.

50. Shang C. Prospective tests on biological models of acupuncture. Evid Based Complement Alternat Med. 2009 Mar; 6(1):31-9.

51. Bowsher D. Mechanisms of acupuncture. In: Filshie J, White A. editors. Medial Acupuncture: a western scientific approach. London: Churchill Livingstone; 1988:69-82.

52. Ma SX. Neurobiology of acupuncture: Toward CAM. Evid Based Complement Alternat Med. 2004 Jun;1(1):41-7.

53. Li J, Wang Q, Liang H, Dong H, Li Y, Ng EH, et al. Biophysical characteristics of meridians and acupoints: a systematic review. Evid Based Complement Alternat Med. 2012;2012793841.

54. Cardeal EL, Oliveira KB, Aoki MN, Amarante MK, Oliveira CEC, Suzuki S, et al. Aspectos imunológicos da acupuntura. Biosaúde 2005 Dez:7(1/2):49-60. 
55. Szabó MVRS, Bechara GH. Acupuntura: Bases Científicas e Aplicações. Ciência rural 2001:31(6):1091-99

56. Saad M. A Medicina tradicional chinesa tem base científica?. Einstein: Educ Contin Saúde 2008: 6(3):122-6.

57. Alvarenga TF, Amaral CG, Steffen CP. Ação da acupuntura na neurofisiologia da dor: revisão bibliográfica. Rev Amazôn Scienc \& Helth 2014 Out/Dez:2(4):29-36.

58. Pereira FAO. Evidências científicas da ação da acupuntura. Perspectivas 2005 jan/jul:4(7):88-105.

59. Taffarel MO, Freitas PMC. Acupuntura e analgesia: aplicações clínicas e principais acupontos. Ciênc Rural 2009 Dez:39(9): 2665-72.

60. Lopes LF, Lopes MC, Fialho FAP, Gonçalves AL. Sistema de conhecimento para diagnóstico em acupuntura:uma modelagem usando o CommonKADS. Gest Prod 2010 Dez:x(x):1-15.

61.Zhu J, Arsovska B, Kozovska K. Acupuncture treatment for sports injury hamstring muscles group, Inter J Clin Exper Med Sci 2017 Nov;3(6):71-3.

62. Vickers AJ, Cronin AM, Maschino AC, Lewith G, MacPherson H, Foster NE, et al. Acupuncture for chronic pain individual patient data meta-analysis, Arch Intern Med 2012 Oct;172(19):1444-53.

63. Toda S. Effect of acupuncture on carnitine for skeletal muscle fatigue, Chin Med 2012 Jan;3:9-12.

64. Lopes SS, Mota MPG. Influência da acupuntura no limiar de percepção dolorosa de musculatura submetida a esforço repetitivo, $\mathrm{Br} \mathrm{J}$ Pain 2018 julset;1(3):207-11.

65. Lee, SH, Chung, SH, Lee, JS, Kim, SS, Shin, HD, Lim, BV, et al. Effects Acupunturaq on the 5-hydroxytryptamine synthesis and tryptophan hydroxylase expression in dorsal in the dorsal raphe of exercice rats. Neurocienses Letters 2002 Oct;332(1): 17-20.

66. Zyloney CE, Jensen K, Polich G, Loiotile RE, Cheetham A, LaViolette PS, et al. Imaging the functional connectivity of the Periaqueductal Gray during genuine and sham electroacupuncture treatment. Mol pain. 2010 Nov;6:80 
67. Minori AET, Mejia DPM. Atuação da Acupuntura para o Tratamento de LER/DORT no Ombro. Goiânia. Monografia [Pós Graduação em Acupuntura] Faculdade Ávila; 2007.

68. Brum KN, Alonso, AC, Brech GC. Tratamento de massagem e acupuntura em corredoresrecreacionais com síndrome do piriforme. Arq Cienc Sau 2009 abrjun; 16(2): 62-6.

69. Hongwen S. Clinical Observation on Acupuncture Treatment of Piriformis Syndrome. J Tradit Chin Med. 2003 mar; 23(1):38-9.

70. Rocha TBX, Vilela Junior GB, Martins GC, Manzatto L, Grande AJ. Análise Comparativa Eletromiográfica do Reto Femoral em Isometria na Posição Inferior do Agachamento Wall Slide, antes e após a Aplicação da Acupuntura no Ponto ST45. Rev Bras Cien e Mov 2012 jul; 20(4):92-8.

71. Pinheiro RG, Mejia DPM. Efeito da Acupuntura na Melhora do Paciente com Quadro Álgico de Lesão de Menisco Medial. Goiânia. Monografia [Pós Graduação em Acupuntura] - Faculdade Ávila; 2012.

72. Nunes EA, Mejia DPM. Tratamento de Acupuntura para Combater Dores nos Ombros. Goiânia. Monografia [Pós Graduação em Acupuntura] - Faculdade Ávila; 2012.

73. Parisotto D. Efeito Imediato da Aplicação da Acupuntura na Dor Muscular Tardia e na Capacidade de Contração Muscular. Curitiba. Dissertação [Mestrado em Fisiologia] - Universidade Federal do Paraná; 2014.

74. Tolentino F. Efeito de um Tratamento comAuriculoterapia na Dor, Funcionalidade e Mobilidade de Adultos com Dor Lombar Crônica. Rio Claro. Dissertação [Mestrado em Desenvolvimento Humano e Tecnologias] Universidade Estadual Paulista - UNESP; 2016.

Enviado: Abril, 2019.

Aprobado: Octubre, 2019. 